\title{
Acquisition of partial grain orientation information using optical microscopy
}

\author{
Matteo Seita*,1, Michael M. Nimerfroh ${ }^{1}$, Michael J. Demkowicz ${ }^{2}$ \\ ${ }^{1}$ Department of Materials Science \& Engineering, Massachusetts Institute of \\ Technology, Cambridge, MA 02139 \\ ${ }^{2}$ Materials Science and Engineering, Texas A\&M University, College Station, TX \\ 77843
}

\begin{abstract}
Optical microscopy (OM) is often the first technique used to characterize surfaces of materials because of its simplicity and low cost. However, the information obtained by $\mathrm{OM}$ is usually limited to the geometry and spatial distribution of microstructural features. We present an advanced OM technique to perform quantitative microstructure analysis of polycrystalline metals and to assess partial grain orientation information from measurements of reflectance over a range of incident light directions. Our technique relies on the collection of a series of optical micrographs under controlled illumination conditions. We find a marked directiondependence of reflectance and ascribe it to sub-micron facets on the surfaces of individual grains. These surface facets are, in turn, correlated to grain orientation. We automate our technique via digital processing and numerical analysis of micrographs, enabling it to generate a wealth of microstructure data while remaining simple to use and inexpensive to implement.
\end{abstract}

\section{Introduction}

Optical microscopes are among the most ubiquitous characterization tools in research laboratories. They are often the simplest and most immediate choice for imaging the surface of many different materials. In polycrystals, optical microscopy (OM) may be used to characterize the geometry and distribution of microstructural features with dimensions of $\sim 1 \mu \mathrm{m}$ and above [1], such as crystal grains [2], precipitates [3], or cracks [4]. However, many elements of polycrystalline microstructures-such as the crystallographic orientations of grains or features with dimensions well below $\sim 1 \mu \mathrm{m}-$ cannot be directly assessed through OM. To characterize such quantities - which are pivotal to interpreting and predicting material properties-researchers usually rely on electron microscopy (EM), even though it is much more resource-intensive than OM $[5,6]$.

We show that computationally-driven, quantitative analysis of surface reflectance enables quantitative assessment of polycrystalline microstructures and markedly extends the range of microstructure information that may be obtained via OM. In particular, we demonstrate the acquisition of partial grain orientation information 
through the characterization of sub-micron surface roughness using OM. While OM cannot replace EM for fundamental microstructure studies, our techniques present a clear advantage over EM for rapid, inexpensive analyses of large surface areas without the need for high vacuum.

Electron backscatter diffraction (EBSD) is an EM-based technique which is routinely employed to perform quantitative metallography measurements [5] and to map grain crystallography [7]. Being able to perform EBSD-like measurements using OM would offer many advantages in the study of materials structure. In fact, capturing an optical micrograph is orders of magnitude faster than acquiring an EBSD scan and requires simpler, less expensive equipment. OM generally has a larger field of view than EM and thus may be used to characterize surfaces of much larger area than EBSD. Moreover, OM measurements may be carried out in water or air and do not require vacuum. Thus, characterization of polycrystalline microstructures by $\mathrm{OM}$ has the potential to enable routine, facile, and high-volume microstructure data acquisition [8]. It may be employed as a non-destructive technique to improve material reliability, for instance by assessing microstructure variability of asprocessed components, or by monitoring the temporal evolution and integrity of ones already in service.

The hallmark of our method is measurement of the intensity of the reflected light as a function of both sample microstructure and incident light direction. For this reason, we name our method directional reflectance microscopy (DRM). We use a custom-made apparatus that allows controlling the light source orientation with respect to the imaged sample and we analyze the collected optical micrographs using MATLAB. We compare our DRM measurements with EBSD analyses using polycrystalline pure nickel (Ni) as a case-study material. We demonstrate that DRM may be used to perform quantitative metallography measurements-such as measurement of grain statistics-as well as to assess microstructural characteristics that are usually only resolvable by electron or X-ray techniques-such as submicron differences in surface roughness and partial crystallographic grain orientation information.

Section 2 describes sample preparation and the development of the apparatus used in our work. The remainder of the paper is divided into two main parts. In the first (section 3), we describe how to construct a DRM dataset and how to use it for quantitative microstructure analysis. We show that grain statistics obtained by DRM are more accurate than ones found by conventional EBSD analysis, in some cases. In the second part (section 4) we demonstrate the capability of DRM to characterize crystallographic orientation of grains by quantifying grain reflectance variations as a function of the incoming light direction. We conclude with a discussion of our findings in section 5 .

\section{Experimental}


The Ni sample used in this study was produced from melting high purity Ni pellets $(99.995 \%)$ into a $30 \mathrm{~g}$ master ingot. A $8 \mathrm{~mm}$ thick disc was cut from the master ingot using wire electrical discharge machining. The disc underwent a series of rolling and annealing cycles until its thickness was reduced to $\sim 1 \mathrm{~mm}$ and it was then cut into a $1.5 \mathrm{~cm} \times 1.8 \mathrm{~cm}$ chip. Each cycle consisted of a $50 \%$ thickness reduction, fine grinding using 1200 grit paper, and annealing at $1300^{\circ} \mathrm{C}$ for 30 minutes in $99.999 \%$ pure Ar. The fine grinding step was used to remove the native oxide film that may form after each cycle and contaminate the rollers. This thermo-mechanical treatment was performed to ensure complete recrystallization of the sample while limiting the formation of crystallographic textures and minimizing work hardening. The annealing temperature was chosen near the melting temperature of $\mathrm{Ni}$ to maximize the kinetics of grain growth as well as to thermally etch the sample surface. After this thermo-mechanical treatment, the sample exhibited clear grain contrast, which is readily visible by $\mathrm{OM}$ without further surface preparation, as shown in Figure 1a.

The sample was mounted onto a 3-D printed circular stage containing 36 markers around the perimeter, equally spaced every $10^{\circ}$. The 3-D printed stage was fixed to a table supporting an Olympus SZ6145 Trinocular Stereozoom optical microscope and was not moved during imaging. A custom-made stainless steel goniometer was fabricated such that it could freely rotate about the 3-D printed stage. This setup is presented in Figure 1b. The goniometer was used to control the incident light direction throughout DRM measurements. It consisted of two arms firmly attached together: one parallel to the table - and thus to the optical image plane-and the other curved out-of-plane around the 3-D printed stage. The out-of-plane arm was designed to support a collimated light source (Cold White Collimated LED for Olympus by Thorlabs) that could be mounted at 11 different positions along the arm - all of which were at the same radial distance from the center of the 3-D printed stage and spaced at approximately $5^{\circ}$ increments, from $15^{\circ}$ to $65^{\circ}$. Every time the position of the light source was changed, the orientation of the source was manually adjusted such that the light-beam axis pointed at the center of the 3-D printed stage (see Fig. 1b).

The sample microstructure was characterized by EBSD as a baseline for comparisons with our DRM measurements. No surface preparation was required for EBSD after the final thermo-mechanical cycle. Eighty EBSD scans of approximately 2 $\mathrm{mm} \times 2 \mathrm{~mm}$ were sequentially acquired with a step size of $10 \mu \mathrm{m}$ over the entire sample surface using a scanning electron microscope (SEM, Philips XL30). The scans were merged into the image shown in Fig. 2 and analyzed with OIM software to assess crystallographic orientation. We used MTex 3.4.1[9] — a free software for the MATLAB environment-to measure grain statistics and to study the grain boundary (GB) network. All OM, EBSD, and MTex data was post-processed and analyzed using MATLAB 2014a.

The surface topography of the Ni sample was characterized by atomic force microscopy (AFM) using a Triboindenter nanoindentation system from Hysitron, 
Inc. A series of $42 \mu \mathrm{m} \times 42 \mu \mathrm{m}$ scans was acquired from selected locations and then analyzed with MATLAB to estimate the root mean square (RMS) surface roughness.

\section{Quantitative microstructure analysis}

In this section, we describe how to perform DRM measurements and how to process the optical micrographs to quantify several microstructural features, including the complete grain boundary network and the grain size distribution.

\subsection{Constructing a DRM dataset}

We use the custom-made apparatus described in the foregoing section to precisely correlate the intensity change of the reflected light—or reflectance $R$-across the sample surface as a function of the incident light direction, $I$. Reflectance is defined as the fraction of incident light reaching the optical microscope after scattering from the sample surface.[10] By rotating the goniometer about the 3-D printed stage holding the sample, the azimuthal component of $I, \varphi$, can be precisely varied from $10^{\circ}$ to $360^{\circ}$, with an angular resolution of $10^{\circ}$. Moreover changing the position at which the light source is mounted along the out-of-plane arm of the goniometer allows varying the elevation component of $I, \theta$, from $15^{\circ}$ to $65^{\circ}$, with an angular resolution of $\sim 5^{\circ}$. The angles $\varphi$ and $\theta$ are schematically shown in Figure $1 \mathrm{~b}$.

We acquire a total of $36 \times 11=396$ optical micrographs of the Ni sample surfaceone for each combination of $\varphi$ and $\theta$-using a CCD camera connected to the microscope. All images are loaded in MATLAB and processed using specially designed algorithms. A detailed description of the algorithms and the analysis performed is presented in the following sections. Three representative $\mathrm{Ni}$ micrographs are reported in Figure 3a. Camera settings such as exposure time, contrast, and brightness are not changed throughout image acquisition. The pixel resolution of the micrographs is $2588 \times 1960$. Because the intensity profile of the collimated light-beam is not uniform, some regions on the sample surface appear brighter than others (see Figure 3a). Moreover, due to the large sample size, some portions of the sample surface (such as the corners) cannot be equally illuminated at all $(\varphi, \theta)$ combinations and thus spuriously appear with dark contrast in some images. To circumvent these difficulties, we also acquire 396 background images of the light-beam intensity profile on the optical image plane as a function of $I$, and then subtract them from the corresponding Ni micrographs. The background images are collected sequentially after each Ni micrograph by laying a large piece of white paper on the sample. Some representative background images are reported in Figure $3 \mathrm{~b}$ for the corresponding Ni micrographs of Figure 3a. Figure $3 \mathrm{c}$ also shows the normalized Ni micrographs after background subtraction. The background subtraction is performed in MATLAB after the background images are processed using a low-pass filter to remove the texture of the white paper used. 
Since the sample was not moved throughout the acquisition of the $396 \mathrm{Ni}$ micrographs, no image registration is required to quantify the change in reflectance across the sample surface as a function of $I$. Moreover, since all Ni micrographs have been normalized through background subtraction, differences in $R$ across the sample surface and for any value of $\varphi$ and $\theta$ can be directly compared. We express the reflectance, $R$, of each pixel with coordinates $(x, y)$ in the Ni micrographs as a function of the incident light direction: $R(x, y, \varphi, \theta)$. Fig. 3 shows clear differences between the reflectance of different grains. Thus, we surmise that $R$ encodes information about the surface structure of individual grains and, through it, about the grain orientations:

$$
R=M(S(g,[a d], T), \varphi, \theta) \quad \text { Eqn. } 1
$$

Here, $M$ is a function that describes the scattering of light as a result of the surface structure $S$ and the incident light direction, $(\varphi, \theta) . S(g,[a d], T)$ describes the surface structure as a function of grain orientation, expressed as the rotation matrix $g$, the chemistry of the surface [11], expressed as a list of adsorbates [ad], and the temperature used during sample preparation [12], $T$. Numerous additional parameters may in general be required to fully predict surface structure. Our goal is to discover the forms of functions $M$ and $S$ and, ultimately, to invert the relationship expressed in Eqn. 1 to obtain grain orientations, $g$, from reflectance, $R$.

Rather than analyzing the change in reflectance of each pixel composing the $\mathrm{Ni}$ micrographs, we segment the images into the sample constituent grains, which we will treat as regions with equal crystal orientation-and thus equal $R$. To segment the Ni micrographs in the sample constituent grains, we first reconstruct the grain boundary (GB) network. This process has been previously employed as a means to assess GB networks in polycrystalline aluminum samples with similar microstructure to the Ni samples used here and is described in detail in another publication [8]. Each Ni micrograph is post-processed using a MATLAB routine that identifies the GB traces using a contrast-based edge detection algorithm [13]. The algorithm detects discontinuities in reflectance larger than a pre-specified threshold across the sample surface. All processed Ni micrographs are then summed into a single cumulative micrograph containing the complete GB network. Here a GB is defined by a crystallographic misorientation of at least $5^{\circ}$, similar to what is used in other techniques [7]. The GB network measured using the foregoing approach is shown in Figure 4. The resulting GB network micrograph consists of a sparse binary matrix, in which only pixels coinciding with a GB trace have logical value 1 . We then take the logical negation of this matrix, which consists of continuous regions of value 1 -corresponding to the sample constituent grains-surrounded by boundaries of value 0 -namely the GB traces (see Fig. $5 a$ ). The new matrix is analyzed using the Image Processing Toolbox in MATLAB 2014a, which automatically returns the centroid coordinates, $\left(x_{C}, y_{C}\right)$, and the area of the "logical grain" (namely the ensemble of pixels with value 1 contained within the grain perimeter) for each detected crystal grain (see Fig. 5a). 
Once each grain's coordinates and area have been identified, we compute the averaged reflectance over each grain, $R^{\operatorname{grain}}(\varphi, \theta)$, for any combination of $\varphi$ and $\theta$ using the $396 \mathrm{Ni}$ micrographs. We plot $R^{\operatorname{grain}}(\varphi, \theta)$ in directional reflectance profiles (DRPs), which are a visual description of how grain reflectance changes as a function of $I$. Each DRP is specific to the corresponding grain. Examples of three different DRPs are shown in Fig. 5. The reflectance is expressed as a percentage of 256 grey levels (where $0 \%$ is 0 and $100 \%$ is 255 ). The scale bar of each DRP presented here is normalized by its maximum reflectance.

Some striking features of DRPs are readily visible in Figure $5 \mathrm{c}$. For instance, each DRP has clear maxima-usually spread within a narrow $\varphi$ range (of $10^{\circ}$ or $20^{\circ}$ ). In most cases there are two maxima with diametrically opposite $\varphi$ values. Some DRPs have maxima with similar intensity (e.g. the DRP of Grain 2 in Fig. 5c), while others have maxima with different intensity (e.g. the DRP of Grain 3 in Fig. 5c). Some DRPs show rather low reflectance across the entire $(\varphi, \theta)$ range (e.g. the DRP of Grain 1 in Fig. 5c). In section 4, we show that through the analysis of DRPs it is possible to assess some microstructural information that would be otherwise not accessible via conventional OM.

\subsection{Grain statistics}

Using the image segmentation described in the foregoing section we can compute the area of each detected grain and calculate the grain size distribution, shown in Figure 6a. The distribution accounts for all grains with diameter larger than $90 \mu \mathrm{m}$. This cut-off is chosen to avoid small surface scratches and irregularities of the sample edges from being erroneously included in the grain statistics. The number of grains found by DRM is 1668 , with an average size is of $\sim 250 \mu \mathrm{m}$. To validate these grain statistics, we compare them to ones obtained by EBSD (also reported in Figure $6 a$ ). The EBSD analysis shows a total of 2814 grains (using the same lower-bound cut-off at $90 \mu \mathrm{m}$ diameter) and an average grain size of $\sim 266 \mu \mathrm{m}$. The difference of $2814-1668=1146$ in the grain count is surprising, since the average grain size and shape of the grain size distribution measured by the two techniques are in good agreement.

To understand the origin of such a large discrepancy, we compare the GB network measured by DRM and by EBSD in Figure 6b. The comparison reveals artifacts in the EBSD measurement, which stem from stitching several individual scans together into the complete EBSD dataset. In fact, the EBSD GB network clearly shows a square grid of stitching artifacts, which follow the border of the eighty individual scans used to characterize the Ni sample. The grid rows and columns are indicated by arrows in Fig. 6b and are also visible in the total EBSD grain map presented in Figure 2. This effect is due to MTEX, which spuriously identifies stitches as GBs because EBSD erroneously suggests grain orientation differences at the square-scan edges, where there is in fact no orientation difference. 
This shortcoming is not due to poor stitching, but to distortion artifacts inherent to EBSD. The distortion-which is referred to as trapezoidal distortion-comes from the non-uniform deflection of the electron beam across the scan and is especially pronounced at low magnifications [14]. Trapezoidal distortion is also the cause of image shearing noticeable when comparing Figures 2 and 4 . A detail of the $\mathrm{Ni}$ sample microstructure showing the effect of trapezoidal distortion is presented in Figure 7 for a few large grains. Because of the distortion, the measured crystallographic orientation may vary by several degrees even across an individual grain with nominally uniform orientation. For instance, in Figure 7 a gradual color change from pink to yellow is visible across a single grain. If the grain happens to be in-between two consecutive EBSD scans-as is the case in Figure 7-one or more spurious GBs will be introduced wherever images are stitched together and a single grain may be split up in two or more smaller grains, introducing an error in the grain count. In the example of Figure 7, the GBs indicated by the arrows measure from $15^{\circ}$ to $18^{\circ}$ in misorientation angle. As a result, the single grain is divided in two.

To estimate the error in the EBSD grain count, we consider that each one of the eighty EBSD square-scans contains roughly 15 spurious grains: twice the scan size $(2 \times 2000 \mu \mathrm{m})$ divided by the average grain size $(266 \mu \mathrm{m})$. Thus the EBSD data set includes about $15 \times 80=1200$ spurious grains, which is very close to the difference of 1146 in the exact grain count between DRM and EBSD.

Intuitively, the distortion-induced effect of "grain splitting" in EBSD should yield a smaller average grain size compared to that measured by DRM. However our measurements show the exact opposite. This unexpected result is due to artificial stretching of the sample area measured by EBSD, which is yet another effect of trapezoidal distortion [14]. By calculating the ratio between sample area obtained by EBSD and DRM, we find that EBSD overestimates the sample area by $15 \%$. Consequently, an improved estimate of the true average grain size may be obtained by dividing $266 \mu \mathrm{m}$ by 1.15 , yielding a value of $\sim 231 \mu \mathrm{m}$, which is smaller than the $\sim 250 \mu \mathrm{m}$ value obtained from DRM. Grain sizes and orientations are not the only quantities affected by trapezoidal distortion: the inclusion of spurious high-angle GBs (i.e. GBs with misorientation angle larger than $15^{\circ}$ ) is expected to also skew GB character distributions. These results show that DRM is more accurate than EBSD at measuring grain and GB statistics of large-area samples because DRM does not distort their areas, does not introduce grain orientation artifacts, and, consequently, does not introduce spurious GBs.

\section{Assessing microstructural information from DRPs}

This section discusses how to use DRPs to characterize the surface structure across the entire polycrystalline sample, infer partial grain orientation information, and 
quantify surface roughness. To this end, we first need to understand the origin of grain reflectance anisotropy.

\subsection{Grain reflectance anisotropy}

The grain contrast seen in the Ni optical micrographs in Fig. 1 and 3 comes from differences in the surface structure of the constituent grains, which are induced by thermal etching $[12,15]$. Since the structure of the thermally reconstructed surfaces depends on their crystallographic orientations, different grains will exhibit terraces and crystallographic facets with distinct orientation, height, and pitch.

Figure 8 shows how the surface structure of three different grains appears in a SEM. Also reported in Figure 8 are the grains' DRPs and inverse pole figures showing their crystallographic orientations (measured by EBSD).

Visual inspection of Figure 8 reveals a strong correlation between the grain surface structure and the orientation and intensity of reflectance maxima in the corresponding DRPs. As may be seen from the electron micrograph, Grain A appears to have a smooth and uniform surface. The corresponding DRP exhibits many, lowintensity reflectance maxima. Conversely, Grain $\mathrm{C}$ shows a much rougher surface with parallel facets that are oriented at an angle $\varphi_{C}=40^{\circ}$ and that are spaced at a distance comparable to their height. The corresponding DRP exhibits two maxima with similar intensities, $R_{1}=45.8 \%$ and $R_{2}=44.3 \%$, at opposite orientations-one at $\varphi_{R_{1}}=310^{\circ}$ (which corresponds to $\varphi_{C}-\pi / 2$ ) and one at $\varphi_{R_{2}}=120^{\circ}$. A different case is that of Grain $B$, whose surface is characterized by facets oriented at an angle $\varphi_{B}=120^{\circ}$ and which are separated by a distance that is visibly greater than their height. For this reason, the facets tend to have one side that is wider than the other. Accordingly, the DRP of Grain B shows two reflectance maxima with very different intensities, $R_{1}=48.2 \%$ and $R_{2}=27.8 \%$. The higher intensity maximum is at $\varphi_{R_{1}}=30^{\circ}$ (which corresponds to $\varphi_{B}-\pi / 2$ ) and the lower intensity maximum is at $\varphi_{R_{2}}=190^{\circ}$.

The interpretation of these measurements comes from simple geometrical considerations, illustrated in Figure 9. The surface facets of a grain may be thought of as an array of stacked "mirrors" that are tilted at an angle, $t$, with respect to the sample surface. Maximum grain reflectance occurs when $I$ is oriented such that specular reflection at the "mirrors" is parallel to the microscope optical axisnamely when the $\varphi$-component of $I$ is perpendicular to the facets' in-plane orientation and the $\theta$-component of $I$ equals $\pi / 2-2 t$ (see Fig. 9). Grains that exhibit facets with same pitch and height, but opposite tilt, such as Grain C in Fig. 8, show two reflectance maxima with opposite $\varphi$ values. Because in this case the facets have sides of comparable size, the opposite maxima have similar intensity. Grains whose facets have one side that is wider than the other-such as Grain B in Fig. 8also show opposite maxima, but with different intensity. Specifically, the largest maximum occurs when $I$ is reflected from the wider sides. The smooth surface that 
characterizes Grain A in Fig. 8 yields a more uniform DRP with no clear maxima. One may argue that $I$ is reflected from Grain A with approximately the same intensity over the entire $(\varphi, \theta)$ range. However, many small reflectance maxima (with intensity up to only a few percent) are still visible in the DRP of Grain A. The largest of these maxima occurs when the $\varphi$-component of $I$ is perpendicular to the side of the shallow facets on the surface of Grain A, which are barely visible from the electron micrograph in Fig. 8. Although small, this signal is clear enough to allow measuring the orientation of such facets, even in a grain as smooth as Grain A. It is indeed remarkable to note that DRM can-indirectly-detect features with size that is below the physical resolution of OM.

\subsection{Assessing partial grain orientation information}

By coloring grains according to the intensities and orientations of reflectance maxima in their DRPs, we can construct "directional reflectance maps," such as those shown in Figure 10. Figure 10a maps a measure of the reflectance of each grain, which is defined as:

$$
R_{\Sigma}=\frac{\left[R_{1}+R_{2}\right]^{\text {grain }}}{\max \left[R_{1}+R_{2}\right]^{\text {sample }}} \cdot 100 .
$$

Here, $\left[R_{1}+R_{2}\right]^{\text {grain }}$ is the sum of the intensities of the two main DRP maxima detected per grain, and $\max \left[R_{1}+R_{2}\right]^{\text {sample }}$ is the maximum among all $\left[R_{1}+\right.$ $R 2$ grain in the sample.

Figure 10b maps the $\varphi$-orientation of the facets in each grain by assessing the $\varphi$ value of their first reflectance maximum, $\varphi_{R_{1}}$, while disregarding its intensity.

To understand the physical meaning of $R_{\Sigma}$ in Fig 10a, we measure the surface roughness of Grains A, B, and C shown in Fig. 8 by means of AFM and we correlate grain roughness to grain reflectance. The results are presented in Figure 11. AFM line scans on the surface of the three grains (not shown here) are analyzed in MATLAB to quantify the height and pitch of the facets. These values are then used to calculate grain roughness in terms of its RMS value. Plotting RMS values versus $R_{\Sigma}$ indeed shows a good correlation (Fig. 11), suggesting that $R_{\Sigma}$ is an indirect measure of grain surface roughness. While this result demonstrates the completely new capability of assessing sub-micron surface roughness by $\mathrm{OM}$, estimating its accuracy and inferring a relationship between grain roughness and reflectance with the form of Eqn. 1 will require more experiments on different materials.

The DRM map in Figure 10b can be used as an EBSD-like grain map to characterize the in-plane orientation of the crystallographic facets at different grains. The colors encode the $\varphi$-coordinate of the facets from $0^{\circ}$ to $170^{\circ}$ with an angular resolution of $10^{\circ}$. 
Since both surface roughness and facet orientation are function of grain crystallography, it is reasonable to expect that DRM maps (and DRPs in general) encode elements of crystallographic orientation of individual grains. While more work is required to precisely decode the relationship between surface facet orientation and grain orientation, our initial results confirm that it is indeed possible to obtain partial crystallographic information from DRM measurements. Figure 8, for instance, suggests that grains with surfaces along $\{101\}$-type planes may have low surface roughness. Figure 12 indeed shows a clear correlation between grains with $R_{\Sigma} \leq 15 \%$ (RMS below $\sim 0.2 \mu \mathrm{m}$ ) and 101 out-of-plane oriented grains measured by EBSD. This finding indicates that DRM maps such as that in Figure 10a may also be used to assess out-of-plane crystallographic textures in polycrystalline metals. However, Figure 13 shows no obvious correlation between the in-plane orientation of the facets (shown in Fig. 10b) and the $\{101\}$ crystallographic planes. We therefore conclude that, while low grain reflectance is a strong indication of 101 out-of-plane orientation, the actual crystallography of the facets is more complex and should be further investigated to infer grain crystallography by means of DRM.

\section{Discussion}

DRM is a promising OM technique to assess the structure and crystallography of polycrystalline materials by quantifying their surface reflectance. At present, DRM may be used to automatically characterize the GB network and the grain size distribution of large planar samples, sometimes with greater accuracy than EBSD. It can also quantify variations in sub-micron surface roughness and infer $\{101\}$ crystallographic textures in pure thermally-etched Ni. However, DRM is materialagnostic and may be used on samples with different surface finish, such as on chemically-etched polycrystalline aluminum [8]. The challenge in broadening the range of materials characterized with DRM is that surface faceting in them may occur differently than described here, requiring additional effort to connect faceting to grain orientation.

The present work investigates samples with flat, chemically clean surfaces. In some cases, surfaces may require special processing before performing a DRM study. For example, surfaces with copious scratches, thick oxide layers, or organic contaminant films may have to be cleaned and polished before the underlying grain structure may be seen. On the other hand, such surfaces may also generate distinct DRPs of their own, which may also be of interest for investigating surface structure and properties. In cases where DRPs contain contributions from both grains and surface imperfections, a thorough understanding of the latter may make it possible to correct for their effect.

Because the automated image analysis performed in DRM returns the complete shape of each grain, DRM may be also used as a high-throughput technique to characterize grain shape descriptors, such as aspect ratios, as well as the curvature of GB traces across the surface of polycrystalline samples. 
All such capabilities may be of interest for a number of applications. For instance, DRM could be employed to characterize rolling textures in FCC materials [16], which are often found to be parallel to the $<101>$ crystallographic direction and affect the material's mechanical behavior. The rapid, high-throughput assessment of surface roughness information makes DRM a candidate technique for estimating the wettability of engineering surfaces $[17,18]$ or for predicting corrosion susceptibility in structural materials [19]. Moreover, the ability to easily characterize GB curvature across the surface of polycrystals could be leveraged both for technological applications and fundamental studies. In fact, as we will show in a separate study [20], GB network maps such as that in Figure 4 can be further analyzed to obtain the distribution of coherent twin boundaries (CTBs) - whose surface traces appear as nearly straight lines [21,22]. CTBs are GBs that have a pronounced effect on material properties: beneficial in some cases-such as imparting mechanical strength and resistance to stress corrosion cracking-[23] and deleterious in others-initiating cracks upon hydrogen charging [24] or under cyclic fatigue [25]. Therefore, being able to assess the distribution of CTBs in a microstructure is of great technological interest for predicting the behavior of polycrystals. On the other hand, monitoring the evolution of GB curvature during thermo-mechanical treatments may also provide fundamental insights into grain growth [26, 27].

While our results have demonstrated a correlation between grain reflectance and grain orientation in a thermally etched Ni sample, unique mapping of reflectance into crystallographic orientation in the form of Eqn. 1 cannot be achieved yet. Part of the reason is because we have limited our analysis of grain reflectance to the intensities and $\varphi$-coordinates of first two DRP maxima. It is reasonable to expect that additional crystallographic orientation information may be obtained by considering all elements of DRPs, such as the number, distribution, spherical coordinates, and angular distance of all maxima (and not only the first two). Moreover, the facets that form on the surface of the different Ni grains may have different crystallography [28]. While the correlation between low surface roughness and $\{101\}$ out-of-plane texture presented in Figure 12 may suggest that grain faceting occurs along $\{101\}$ crystal planes, the lack of a correlation in Figure 13 indicates that grain facets consist of different crystallographic planes, depending on grain orientation. We also expect that using a different etching method (e.g. chemical etching as opposed to thermal etching) may lead to a different correlation between facet crystallography and grain orientation. All these details complicate significantly the solution of Eqn. 1.

One possible approach to find a correlation between grain reflectance and orientation is to develop a "directional reflectance model". In future publications we will propose one candidate model consisting of two components: the first to predict reflectance from surface structure, the second to predict surface structure from grain orientation. The reflectance-structure component of the model will be based on the bidirectional reflectance distribution function (BRDF) [29-31], which describes how light is reflected as a function of surface structure and $I$. BRDF has 
been extensively applied to remote sensing applications as a means to interpret the surface structure of the Earth from measurements of reflectance [32-35], or employed in computer graphics to simulate the appearance of different materials under different illumination conditions [36, 37]. Some early studies have already proposed geometrical models based on BRDF to correlate surface morphology of metallic surfaces to their reflectance [38, 39]. In such models, surface reflectance is expressed as a combination of specular reflection plus diffuse scattering (coming from multiple reflections) as a function of surface faceting and $I$.

The structure-orientation component of the model will leverage the great body of literature that is focused on the study of surface structure reconstruction [40] as a function of crystallography, processing temperature $[12,15]$, and composition of the surrounding medium $[11,41]$. By knowing the orientation of the crystal grains, the crystallography of the material analyzed, and the process used to etch the surface, it may be possible to predict the crystallography of the facets that are expected to form on different grains with varying processing histories and under different conditions. Facet geometry and distribution will then be inferred by accounting for the symmetries imposed by the underlying material crystallography and the surface energies of the different crystal planes.

Following this approach, the directional reflectance model will first predict the surface structure of crystalline grains and then use this information to simulate the corresponding DRPs. It may also be possible to infer grain orientation by comparing features in measured DRPs with a database of simulated ones, similar to how EBSD compares features in measured Kikuchi patterns with look-up tables calculated in advance for the different crystal structures [42].

\section{Acknowledgments}

The authors would like to acknowledge A. Lai for carrying our AFM measurements, I. McCue for sample preparation, and M. Tarkanian for manufacturing the goniometer used for DRM measurements. This work was supported by the US Department of Energy, Office of Basic Energy Sciences under Award No DESC0008926. Access to shared experimental facilities was provided by the MIT Center for Materials Science Engineering.

\section{Figure Captions}

Figure 1: (a) Optical micrograph of the Ni sample used in this study. (b) Photograph of the appartus used to control the incident light direction.

Figure 2: Complete inverse pole figure grain map of the Ni sample used in this study. Grain orientation is visualized along the sample normal direction. The total map consists of eighty EBSD scans that have been merged together using OIM software. 
Figure 3: (a) Three optical micrographs taken under different incident light directions. (b) Corresponding background images showing the non-uniformity of the intensity profile at the optical image plane. They show a brighter halo around the beam-light center and regions that are not illuminated at the edges of the micrographs. (c) The same Ni optical micrographs as in (a) after background subtraction.

Figure 4: Reconstructed GB network using the entire sequence of 396 micrographs.

Figure 5: (a) A portion of the GB network shown in Fig. 4, after logical negation. Three representative grains (in green, red, and blue) are considered to illustrate the process by which DRPs are calculated. The area of the red grain is shown around the grain centroid. (b) Appearance of the red grain in three different micrographs (taken at different combinations of $\boldsymbol{\varphi}$ and $\boldsymbol{\theta}$ ) and corresponding reflectance values $\boldsymbol{R}^{\text {grain }}$ averaged over the convex image. (c) DRPs for the three grains indicated in (a).

Figure 6: (a) Grain size distribution and (b) GB network as measured by DRM and EBSD.

Figure 7: Comparison between DRM and EBSD images of GB networks from the same sample area. The EBSD measurement shows spurious GBs (some of which are indicated by arrows) that arise from stitching of EBSD images with trapezoidal distortion. The EBSD grain map also shows the change in grain color-and thus orientation-from left (pink) to right (yellow) across an individual grain.

Figure 8: Correlation between DRPs, surface structure topography (imaged by SEM) and crystallographic orientation (measured by EBSD) from three different grains. The two largest reflectance intensity maxima are reported in each DRP.

Figure 9: Schematic illustration of grain reflectance dependence on surface morphology. In this example, which is representative of Grain B in Fig. 8, the reflectance maxima $\boldsymbol{R}_{\mathbf{1}}$ and $\boldsymbol{R}_{\mathbf{2}}$ have opposite $\boldsymbol{\varphi}$ value and different intensity: $R_{1}>R_{2}$.

Figure 10: (a) Grain reflectance map compiled considering $\boldsymbol{R}_{\boldsymbol{\Sigma}}$ of each grain. This map provides an indirect characterization of surface roughness. (b) Reflectance directionality map compiled considering only $\boldsymbol{\varphi}_{R_{1}}$ of each grain. This map is an indirect measure of surface facet orientations.

Figure 11: Correlation between surface roughness and reflectance for the three grains shown in Fig. 8. Surface roughness information is reported in terms of facet pitch, height, and RMS (measured by AFM). Grain reflectance is compiled using $\boldsymbol{R}_{\boldsymbol{\Sigma}}$. The correlation is demonstrated by plotting RMS versus $\boldsymbol{R}_{\boldsymbol{\Sigma}}$.

Figure 12: Comparison between the distribution of grains with low surface roughness $\left(\boldsymbol{R}_{\boldsymbol{\Sigma}} \leq \mathbf{1 5} \%\right.$, RMS $\left.\leq 0.2 \mu \mathrm{m}\right)$ and the $\{101\}$ out-of-plane texture in the $\mathrm{Ni}$ sample as measured by EBSD. 
Figure 13: Comparison between the distribution of grains with facets oriented along $\varphi_{R_{1}}=\mathbf{9 0}^{\circ}$ and the $\{101\}$-type crystallographic planes along the same direction as measured by EBSD.

\section{References}

[1] J. M.R. Louthan. Optical Metallography, ASM Handbook Volume 10, Materials Characterization (ASM International) (1986) 299 - 308.

[2] P.L.O. Rossi, C.M. Sellars. Quantitative metallography of recrystallization, Acta Mater. 45 (1997) 137-148.

[3] A. Szczotok. Quantitative evaluation of carbides in nickel-base superalloy MAR-M247, Iop Conf Ser-Mat Sci 22 (2011).

[4] H.E. Exner. Quantitative metallography in three dimensions, Prakt MetallogrPr M 38 (2001) 370-382.

[5] F.J. Humphreys. Quantitative metallography by electron backscattered diffraction, Journal of Microscopy-Oxford 195 (1999) 170-185.

[6] E.F. Rauch, J. Portillo, S. Nicolopoulos, D. Bultreys, S. Rouvimov, P. Moeck. Automated nanocrystal orientation and phase mapping in the transmission electron microscope on the basis of precession electron diffraction, Z Kristallogr 225 (2010) 103-109.

[7] B.L. Adams, S.I. Wright, K. Kunze. Orientation Imaging - the Emergence of a New Microscopy, Metall Trans A 24 (1993) 819-831.

[8] M. Seita, M. Volpi, S. Patala, I. McCue, C.A. Schuh, M.V. Diamanti, J. Erlebacher, M.J. Demkowicz. A high-throughput technique for determining grain boundary character non-destructively in microstructures with through-thickness grains, Npj Computational Materials 2 (2016) 16016.

[9] F. Bachmann, R. Hielscher, H. Schaeben. Texture Analysis with MTEX - Free and Open Source Software Toolbox, Sol St Phen 160 (2010) 63-68.

[10] P. Weightman, D.S. Martin, R.J. Cole, T. Farrell. Reflection anisotropy spectroscopy, Rep Prog Phys 68 (2005) 1251-1341.

[11] H. Minoda, K. Yagi, F.J.M.Z. Heringdorf, A. Meier, D. Kahler, M.H. von Hoegen. Gold-induced faceting on a Si(001) vicinal surface: Spot-profile-analyzing LEED and reflection-electron-microscopy study, Phys. Rev. B 59 (1999) 2363-2375.

[12] W.W. Mullins. Theory of Linear Facet Growth during Thermal Etching, Philos Mag 6 (1961) 1313-1341.

[13] J. Canny. A Computational Approach to Edge Detection, IEEE Transactions on Pattern Analysis and Machine Intelligence PAMI-8 (1986) 679-698.

[14] G. Nolze. Image distortions in SEM and their influences on EBSD measurements, Ultramicroscopy 107 (2007) 172-183.

[15] A.J.W. Moore. The Influence of Surface Energy on Thermal Etching, Acta Metall Mater 6 (1958) 293-304. 
[16] J. Hirsch, K. Lucke. Mechanism of Deformation and Development of Rolling Textures in Polycrystalline Fcc Metals .1. Description of Rolling Texture Development in Homogeneous Cuzn Alloys, Acta Metall Mater 36 (1988) 2863-2882. [17] K.J. Kubiak, M.C.T. Wilson, T.G. Mathia, P. Carval. Wettability versus roughness of engineering surfaces, Wear 271 (2011) 523-528.

[18] A. Shastry, M.J. Case, K.F. Bohringer. Engineering surface roughness to manipulate droplets in microfluidic systems, Proc leee Micr Elect (2005) 694-697. [19] T. Souier, M. Chiesa. Effect of surface conditions and strain hardening on the passivity breakdown of 304 stainless steel, J Mater Res 27 (2012) 1580-1588.

[20] M. Seita, J.P. Hanson, S. Gradečak, M.J. Demkowicz. Probabilistic failure criteria for individual microstructural elements:

an application to hydrogen-assisted crack initiation in alloy 725, Under Review (2016).

[21] V. Randle, H. Davies. A comparison between three-dimensional and twodimensional grain boundary plane analysis, Ultramicroscopy 90 (2002) 153-162.

[22] H. Davies, V. Randle. Single-section plane assessment in grain boundary engineered brass, Journal of Microscopy-Oxford 205 (2002) 253-258.

[23] V. Randle. Twinning-related grain boundary engineering, Acta Mater. 52 (2004) 4067-4081.

[24] M. Seita, J.P. Hanson, S. Gradecak, M.J. Demkowicz. The dual role of coherent twin boundaries in hydrogen embrittlement, Nat. Commun. 6 (2015).

[25] M.D. Sangid, H.J. Maier, H. Sehitoglu. The role of grain boundaries on fatigue crack initiation - An energy approach, Int J Plasticity 27 (2011) 801-821.

[26] E.A. Holm, S.M. Foiles. How Grain Growth Stops: A Mechanism for GrainGrowth Stagnation in Pure Materials, Science 328 (2010) 1138-1141.

[27] E.R. Homer, E.A. Holm, S.M. Foiles, D.L. Olmsted. Trends in Grain Boundary Mobility: Survey of Motion Mechanisms, Jom-Us 66 (2014) 114-120.

[28] H. Meltzman, D. Chatain, D. Avizemer, T.M. Besmann, W.D. Kaplan. The equilibrium crystal shape of nickel, Acta Mater. 59 (2011) 3473-3483.

[29] J.E. Proctor, P.Y. Barnes. NIST high accuracy reference reflectometerspectrophotometer, J Res Natl Inst Stan 101 (1996) 619-627.

[30] Nicodemu.Fe. Reflectance Nomenclature and Directional Reflectance and Emissivity, Appl Optics 9 (1970) 1474-\&.

[31] P.Y. Barnes, E.A. Early, A.C. Parr. Spectral Reflectance, NIST Special Publication, US Government Printing Office 250-48 (1998).

[32] W. Lucht, C.B. Schaaf, A.H. Strahler. An algorithm for the retrieval of albedo from space using semiempirical BRDF models, Ieee T Geosci Remote 38 (2000) 977998.

[33] S.G. Warren, R.E. Brandt, P.O. Hinton. Effect of surface roughness on bidirectional reflectance of Antarctic snow, J Geophys Res-Planet 103 (1998) 2578925807.

[34] J.P. GastelluEtchegorry, V. Demarez, V. Pinel, F. Zagolski. Modeling radiative transfer in heterogeneous 3-D vegetation canopies, Remote Sens Environ 58 (1996) 131-156. 
[35] A. Morel, D. Antoine, B. Gentili. Bidirectional reflectance of oceanic waters: accounting for Raman emission and varying particle scattering phase function, Appl Optics 41 (2002) 6289-6306.

[36] K.J. Dana, B. Van Ginneken, S.K. Nayar, J.J. Koenderink. Reflectance and texture of real-world surfaces, Acm T Graphic 18 (1999) 1-34.

[37] H.P.A. Lensch, J. Kautz, M. Goesele, W. Heidrich, H.P. Seidel. Image-based reconstruction of spatial appearance and geometric detail, Acm T Graphic 22 (2003) 234-257.

[38] J.G. Burnell, J.V. Nicholas, D.R. White. Scattering Model for Rough Oxidized Metal-Surfaces Applicable to Radiation Thermometry of Reformer Furnaces, Opt Eng 34 (1995) 1749-1755.

[39] K.E. Torrance, E.M. Sparrow. Theory for Off-Specular Reflection from Roughened Surfaces, J Opt Soc Am 57 (1967) 1105-\&.

[40] K. Oura. Surface science : an introduction, Springer, Berlin; New York, 2003. [41] X. Bao, G. Lehmpfuhl, G. Weinberg, R. Schlogl, G. Ertl. Variation of the Morphology of Silver Surfaces by Thermal and Catalytic Etching, J Chem Soc Faraday T 88 (1992) 865-872.

[42] A.J. Schwartz, M. Kumar, B.L. Adams. Electron backscatter diffraction in materials science, Kluwer Academic, New York ; London, 2000. 
a.

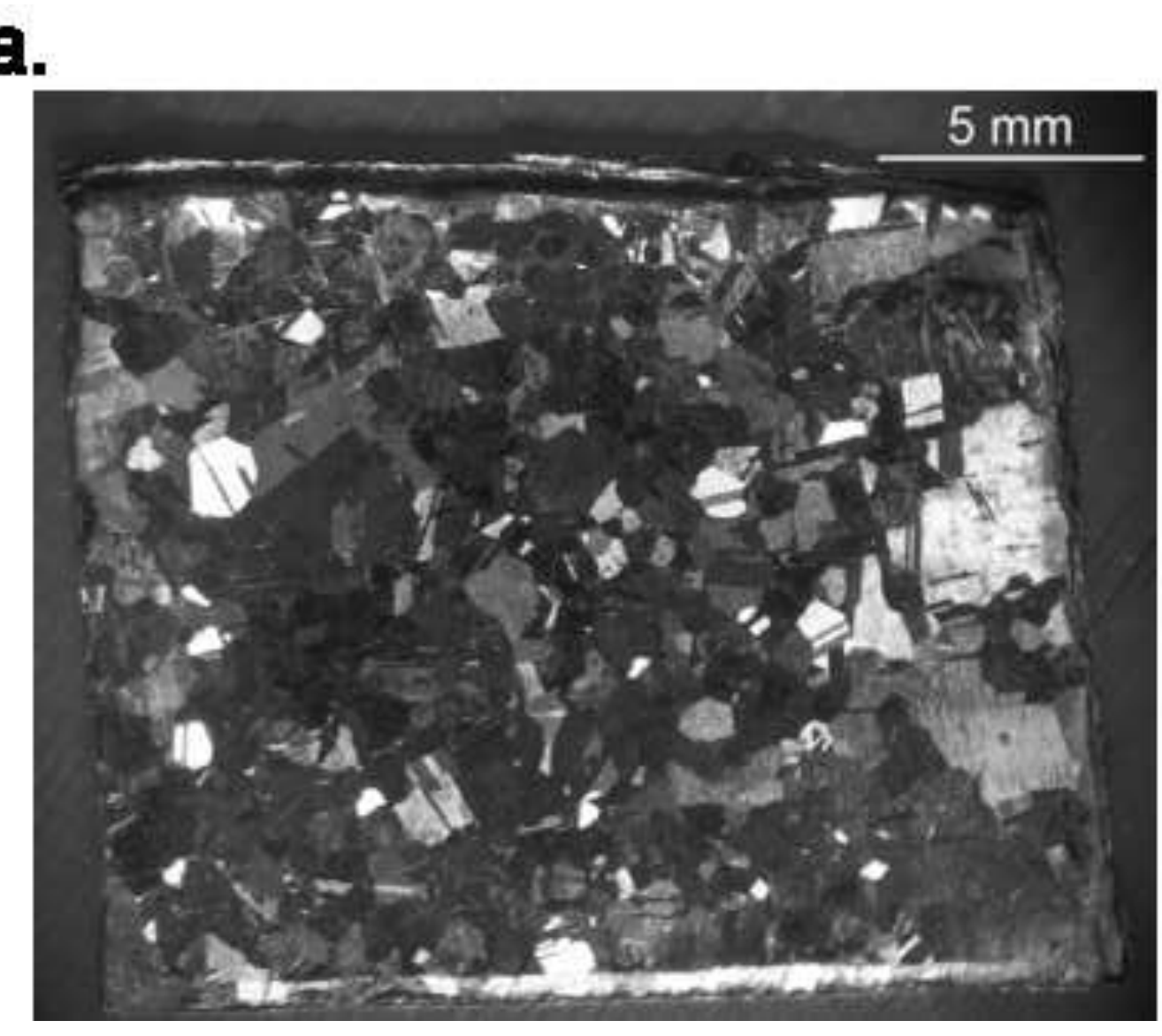

b.

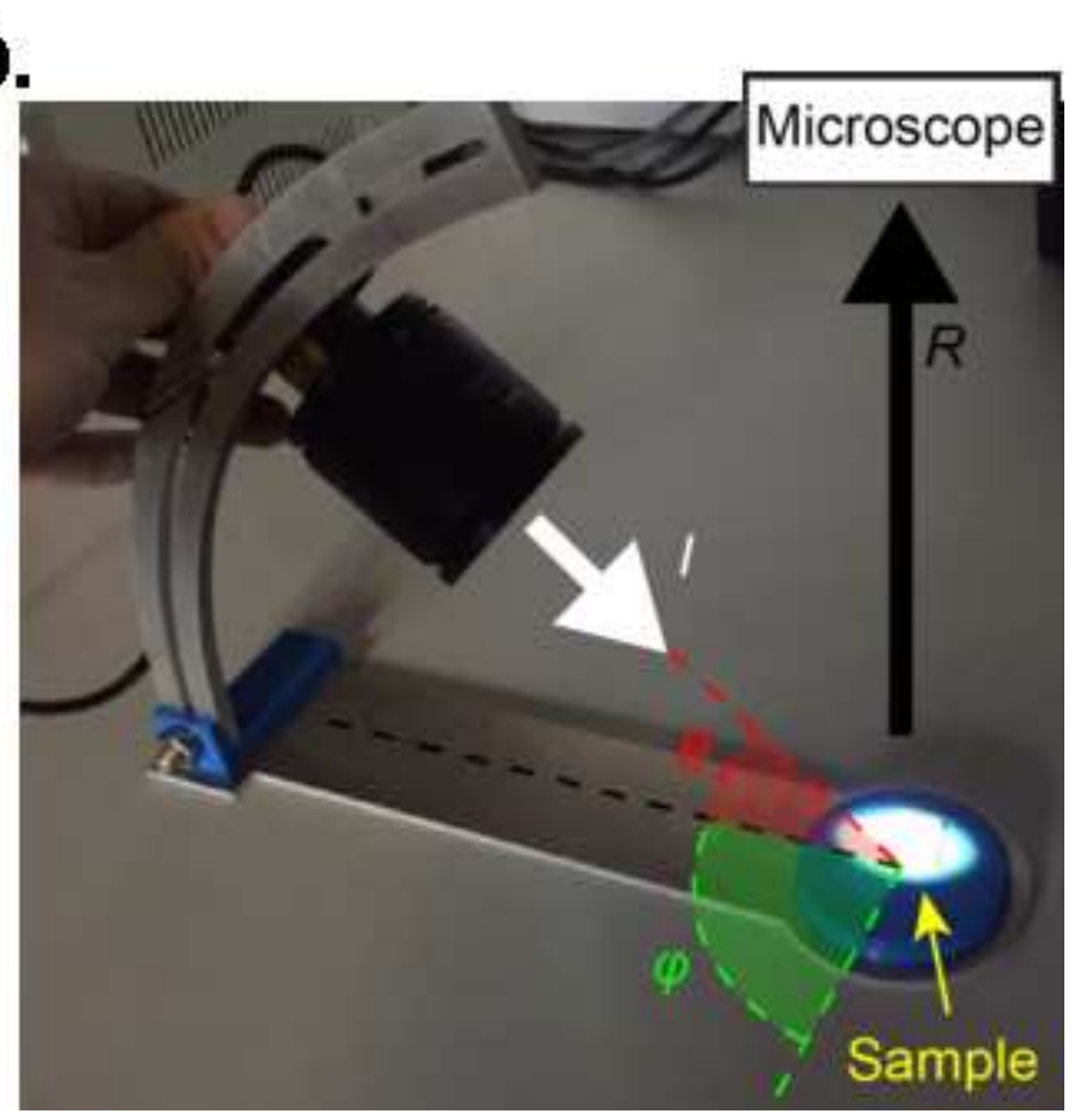




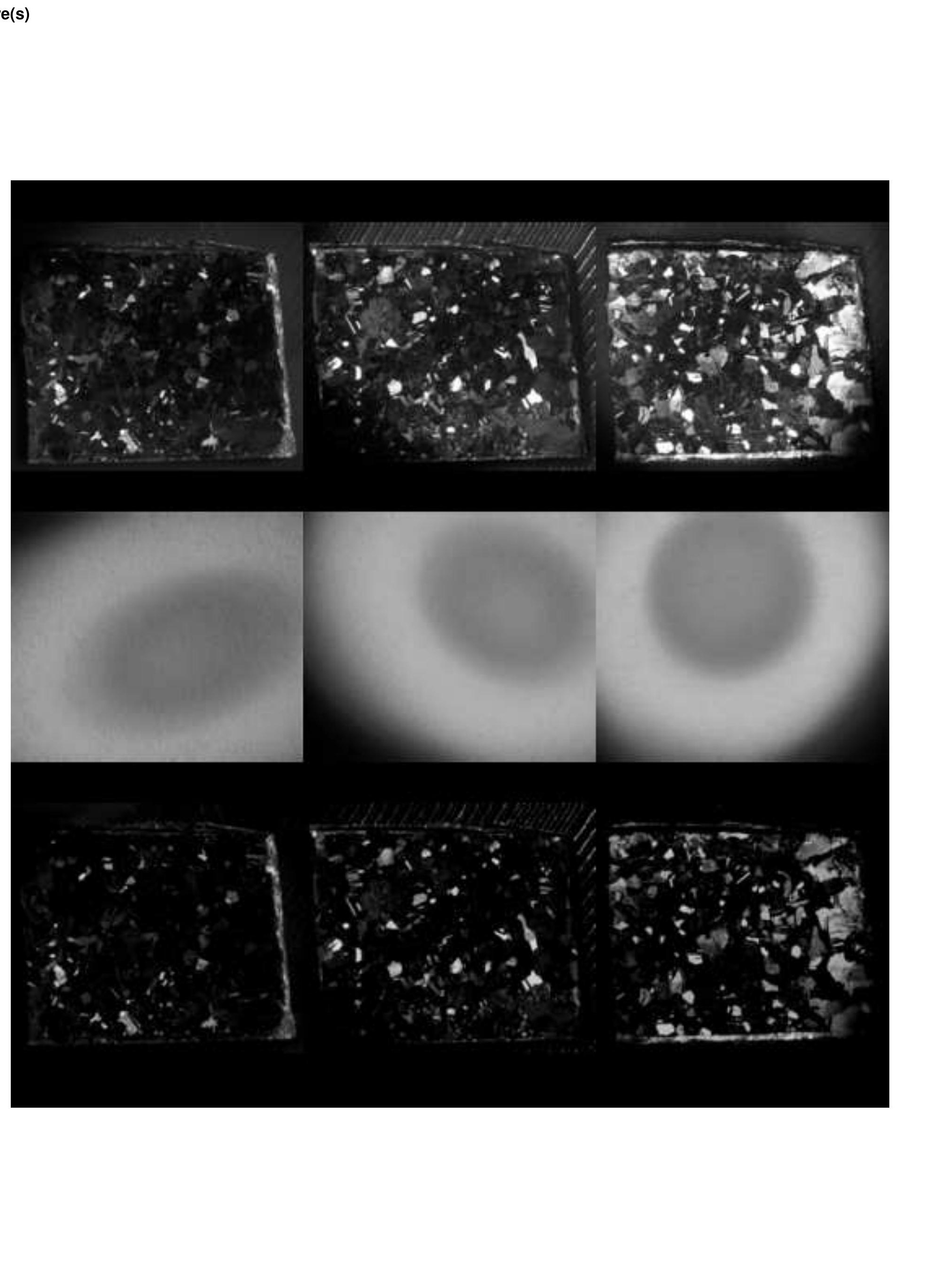

Figure(s)

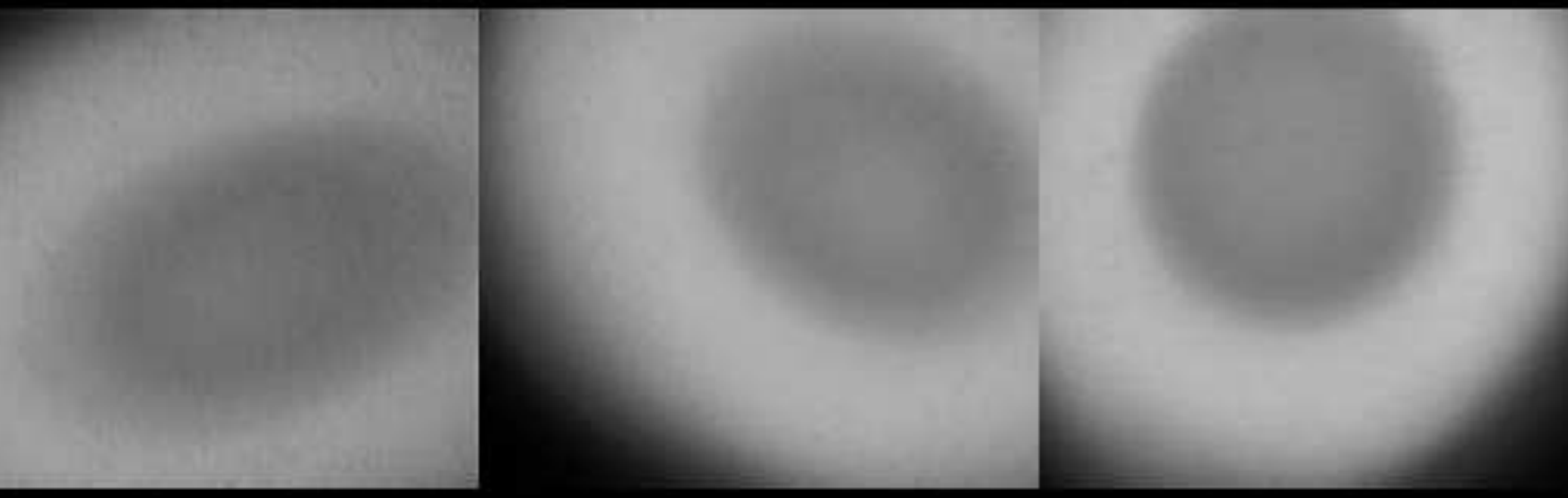
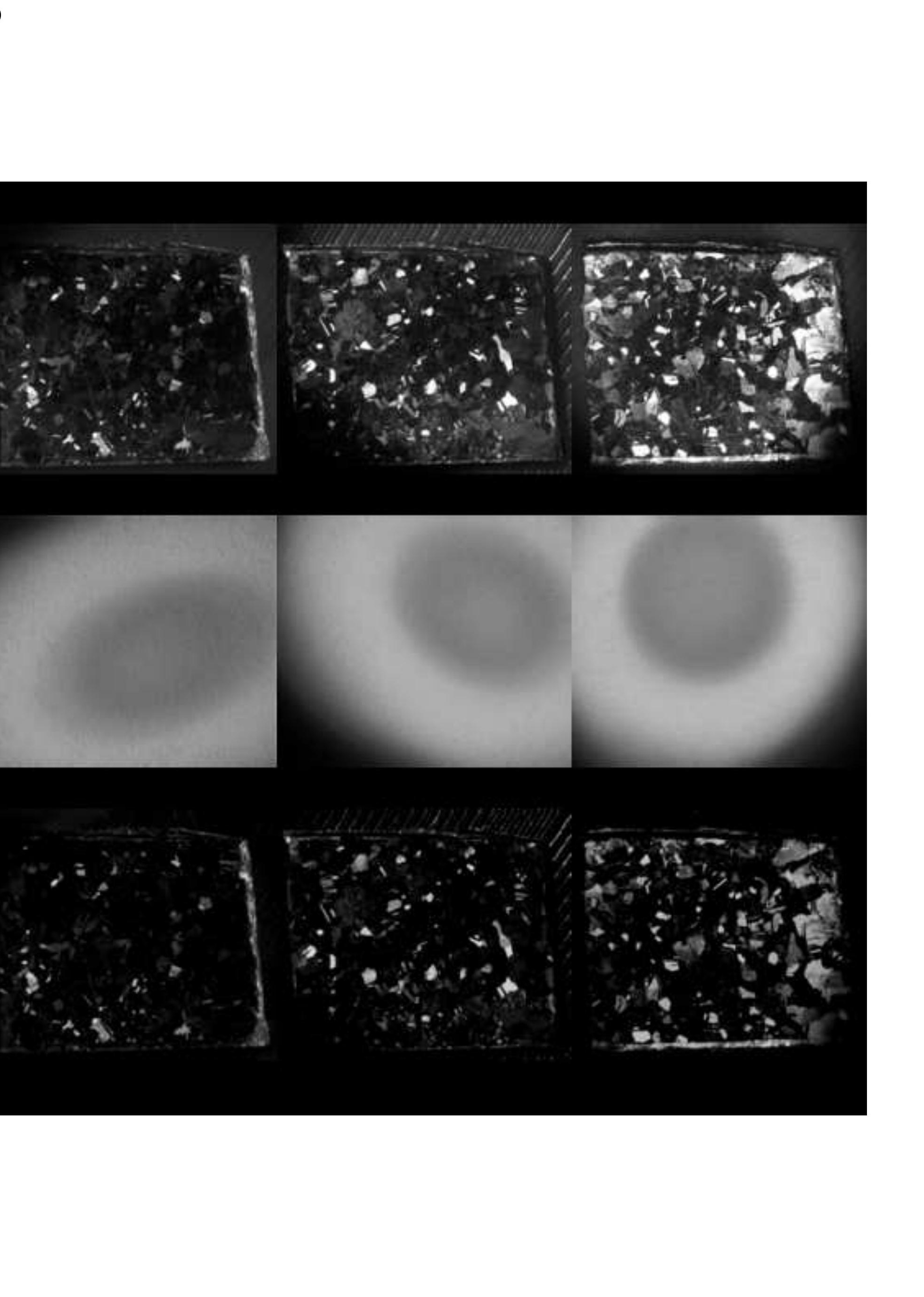


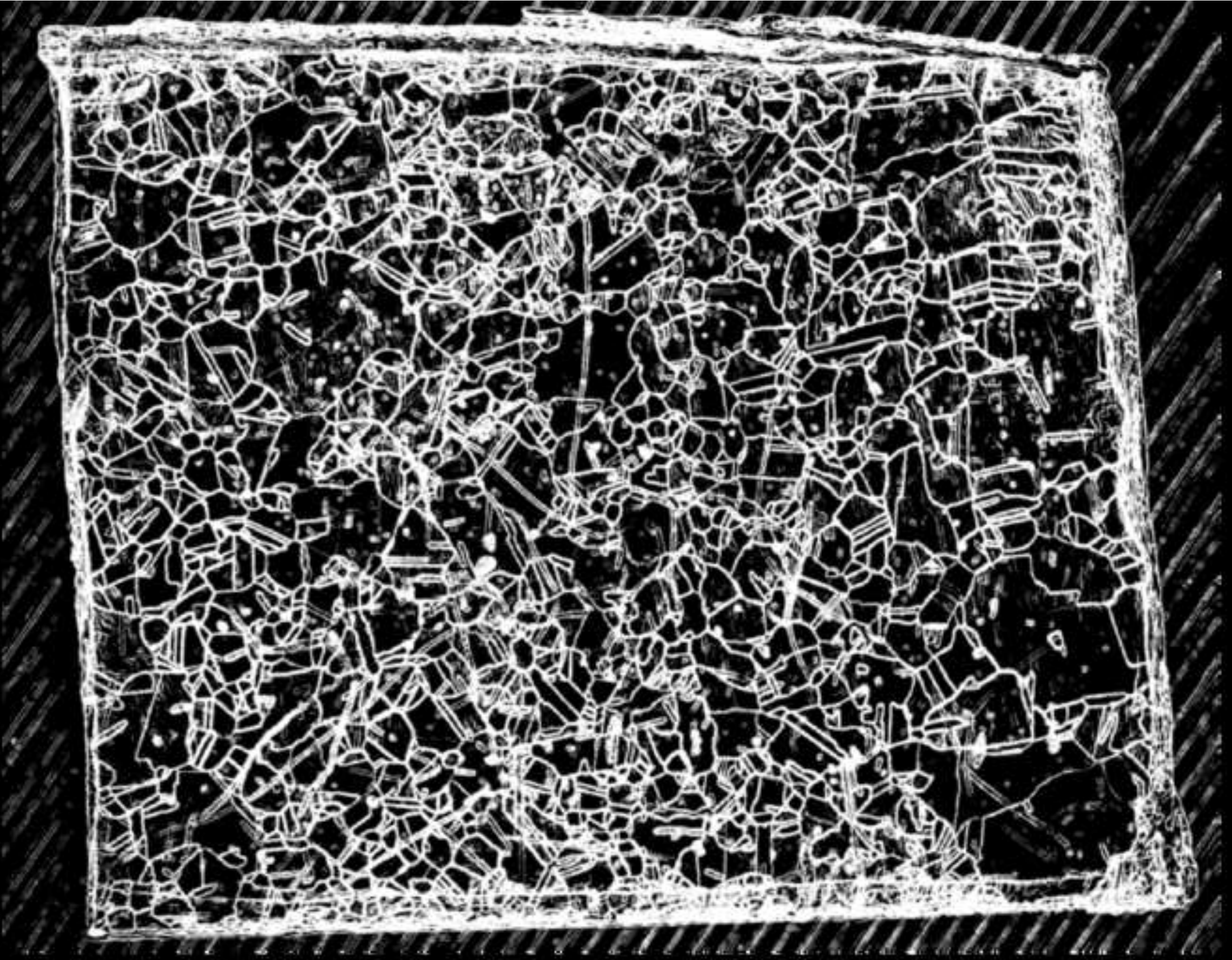


a.

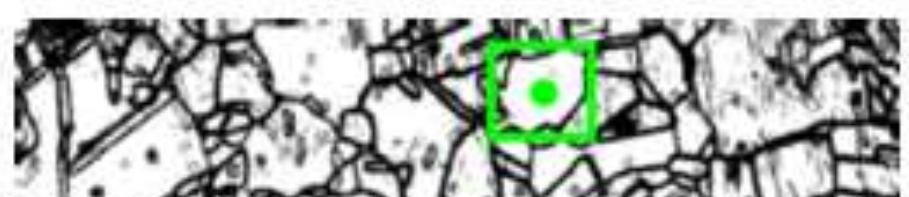

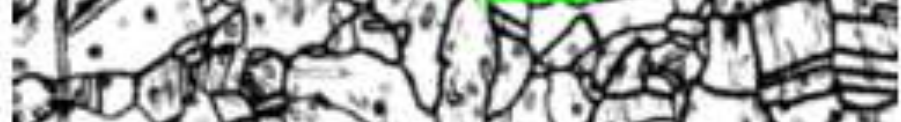

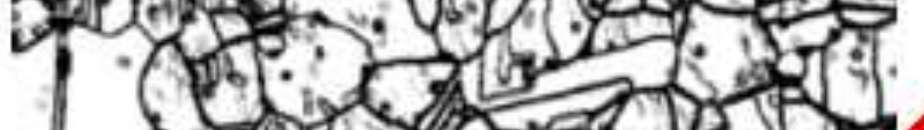
$1 \% 50$ ?

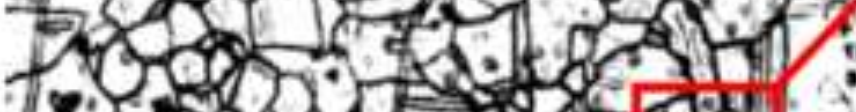

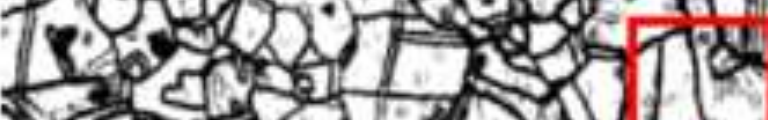

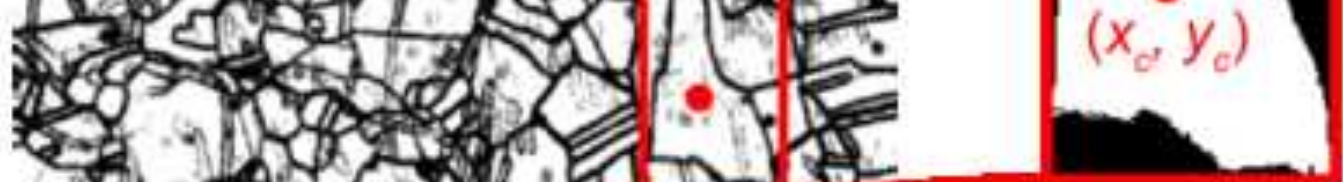

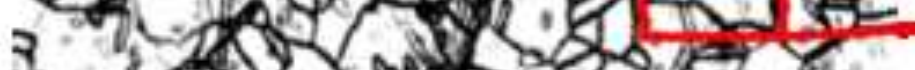

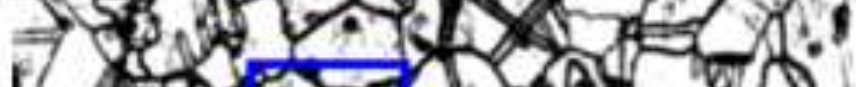
The $\rightarrow$ a d a

b.

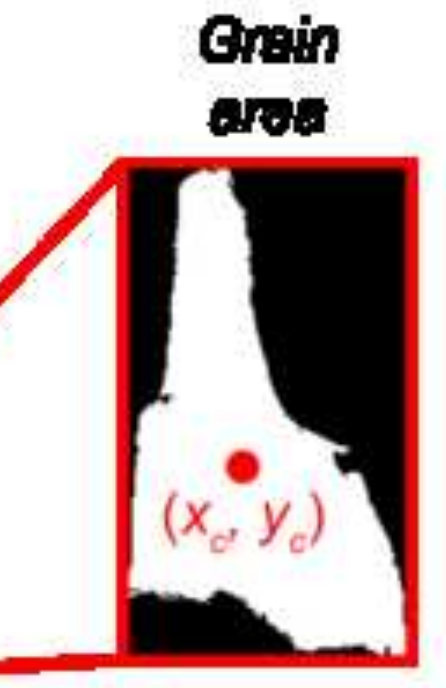

c.
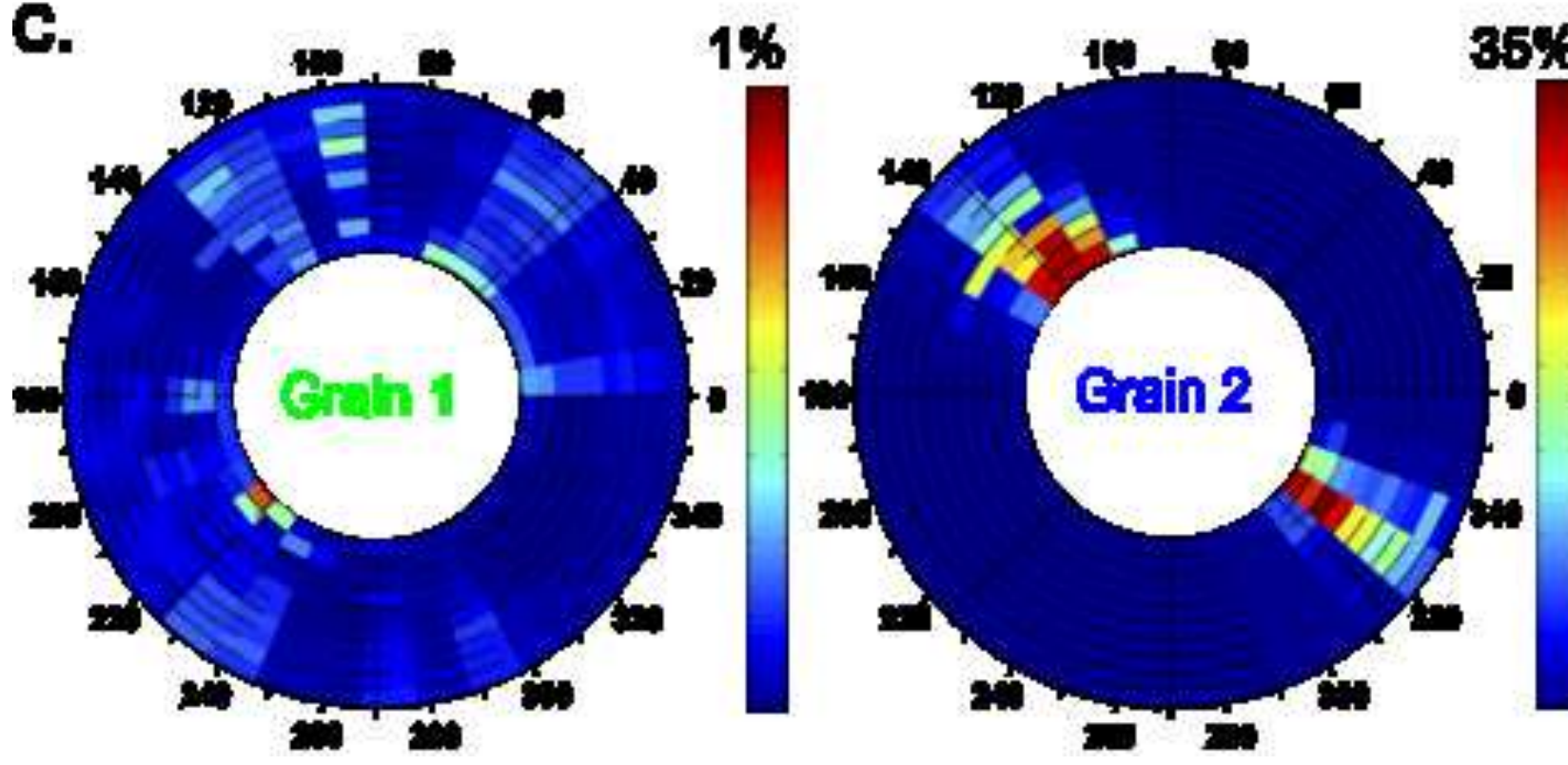

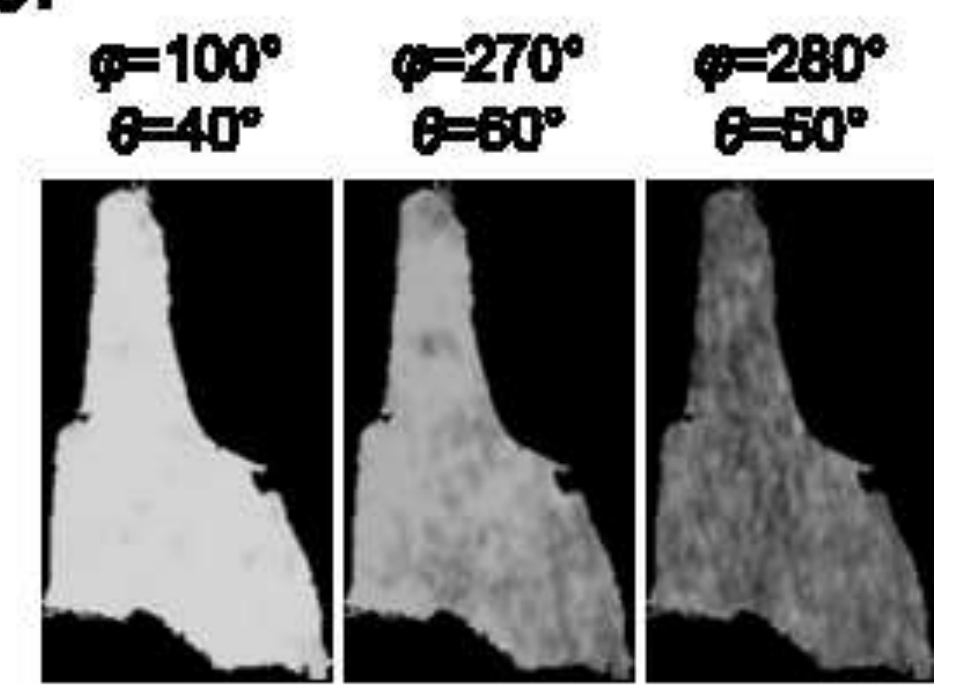

R-39\%

$30 \%$

$19.5 \%$

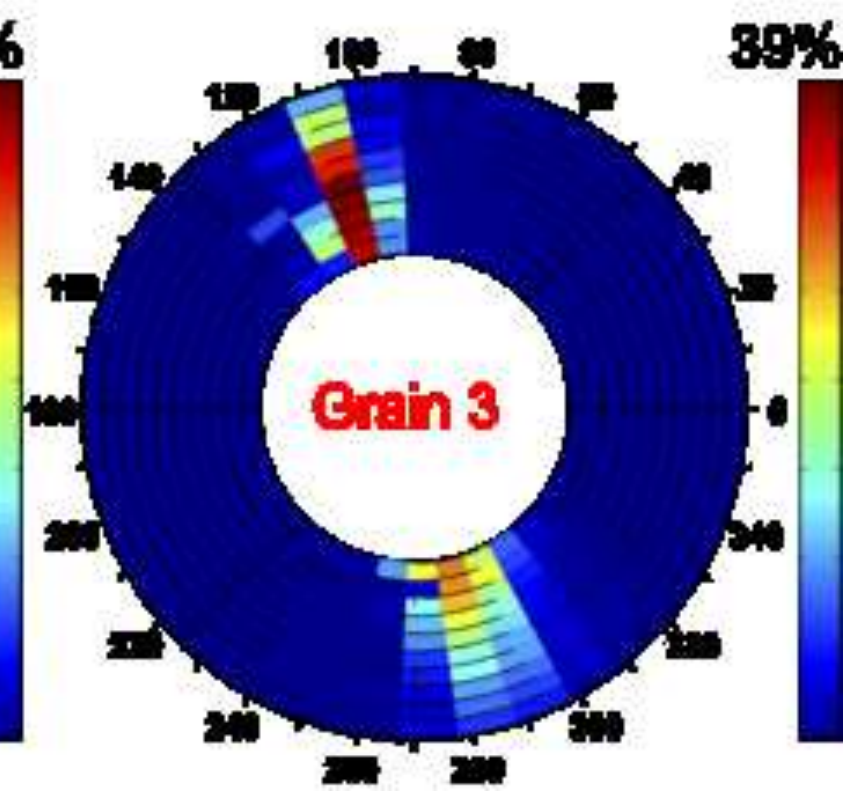


a.

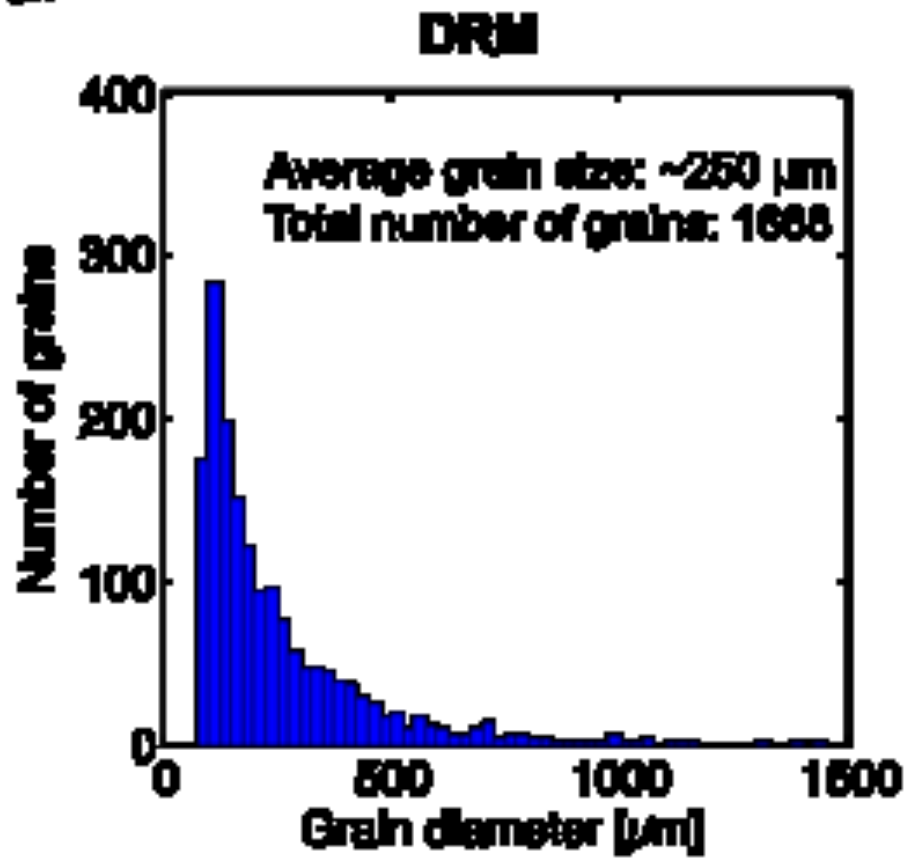

b.

Did

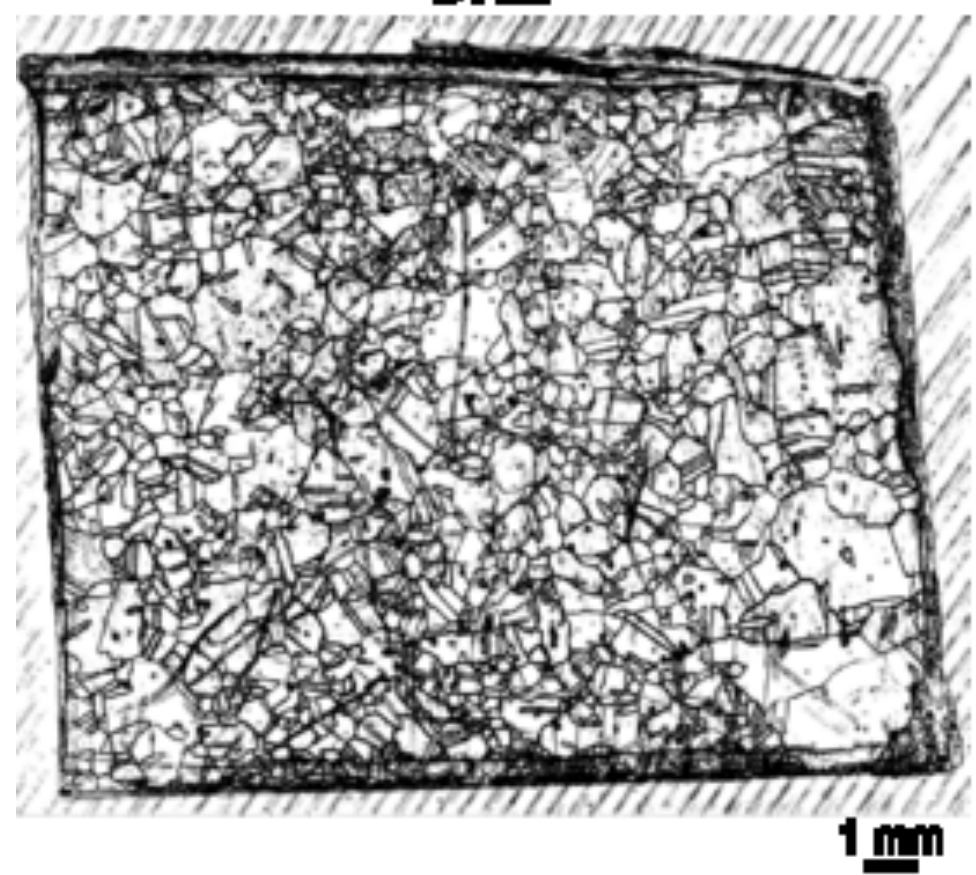

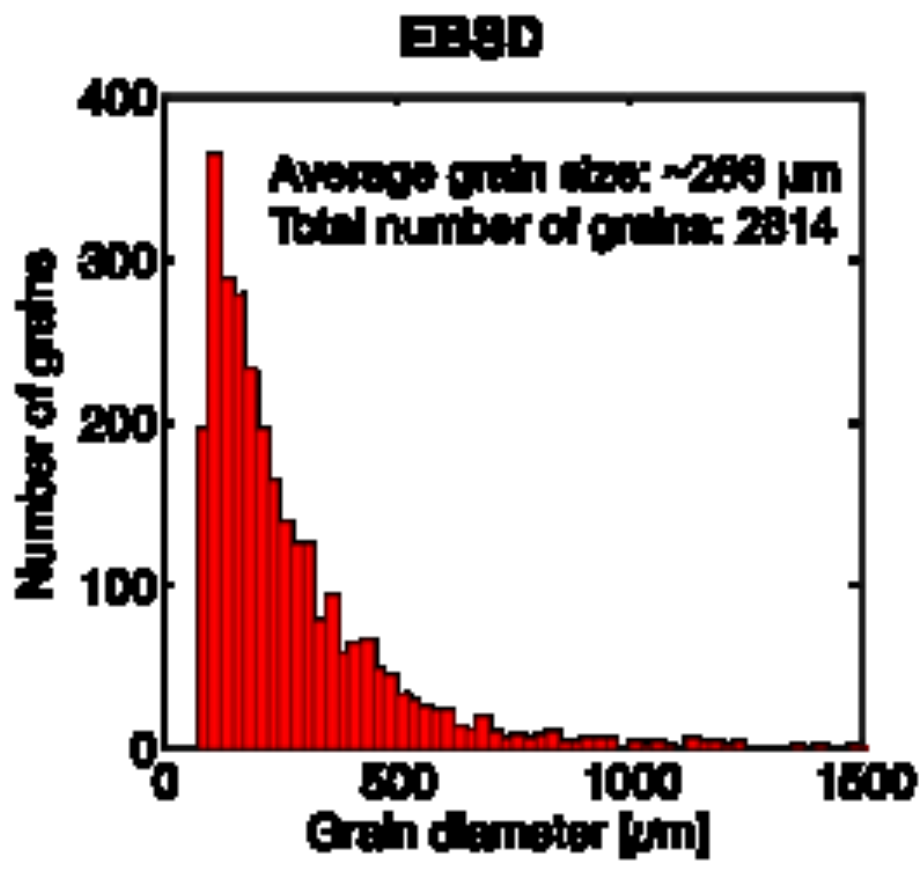

ㄴ:p

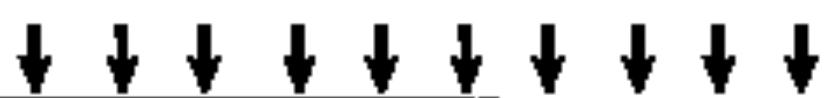

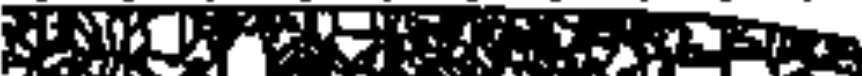
Sh

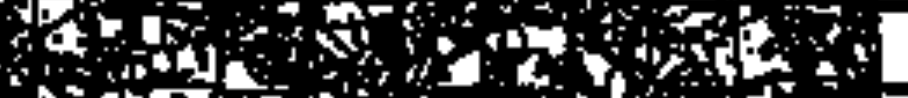

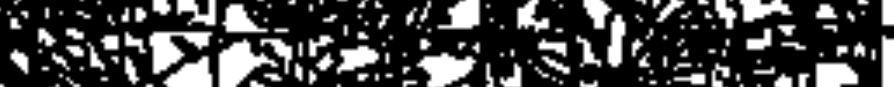
L 2 (n) and ito 12. $1 \mathrm{mn}$ 


\section{DRM}
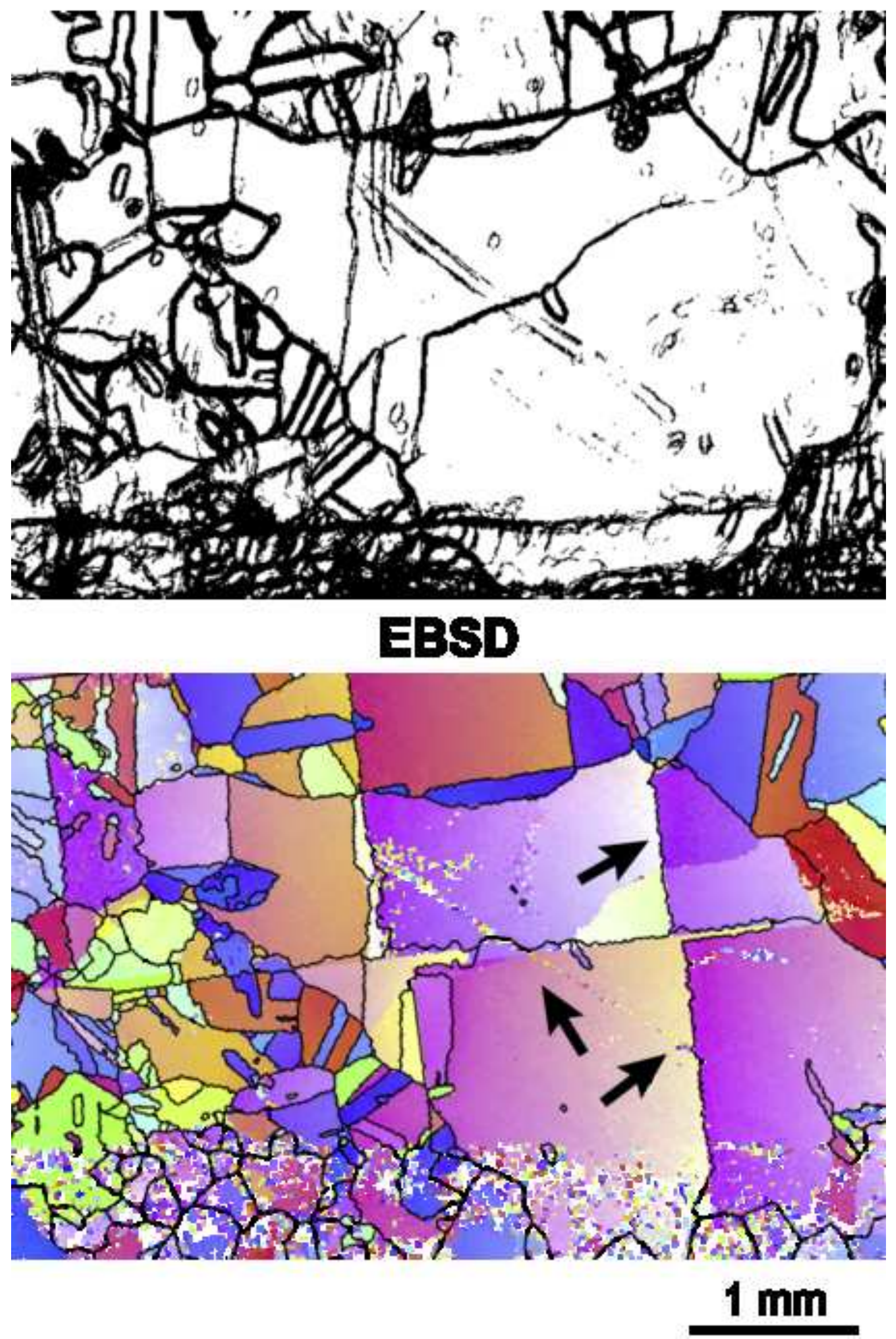

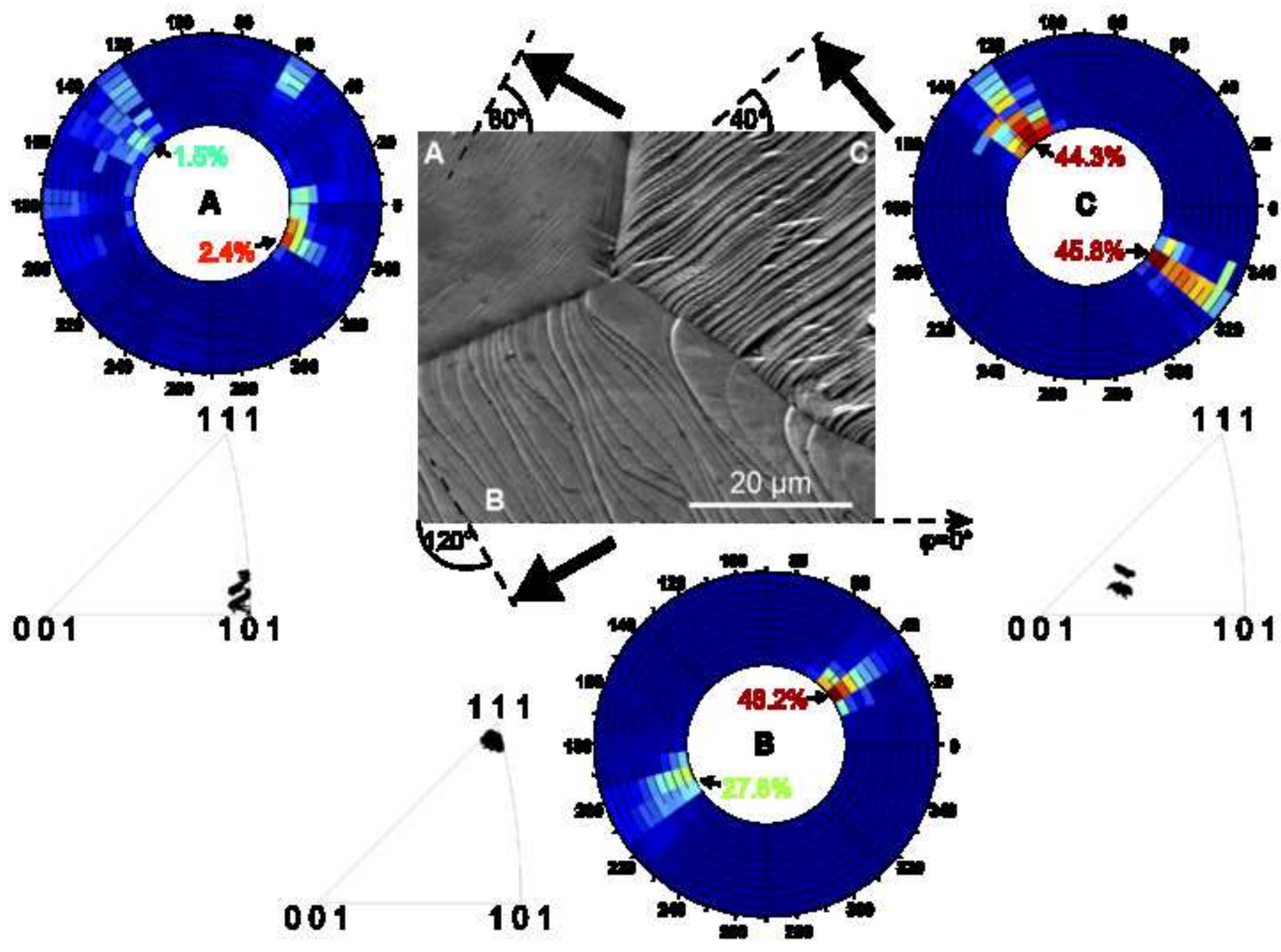

001 


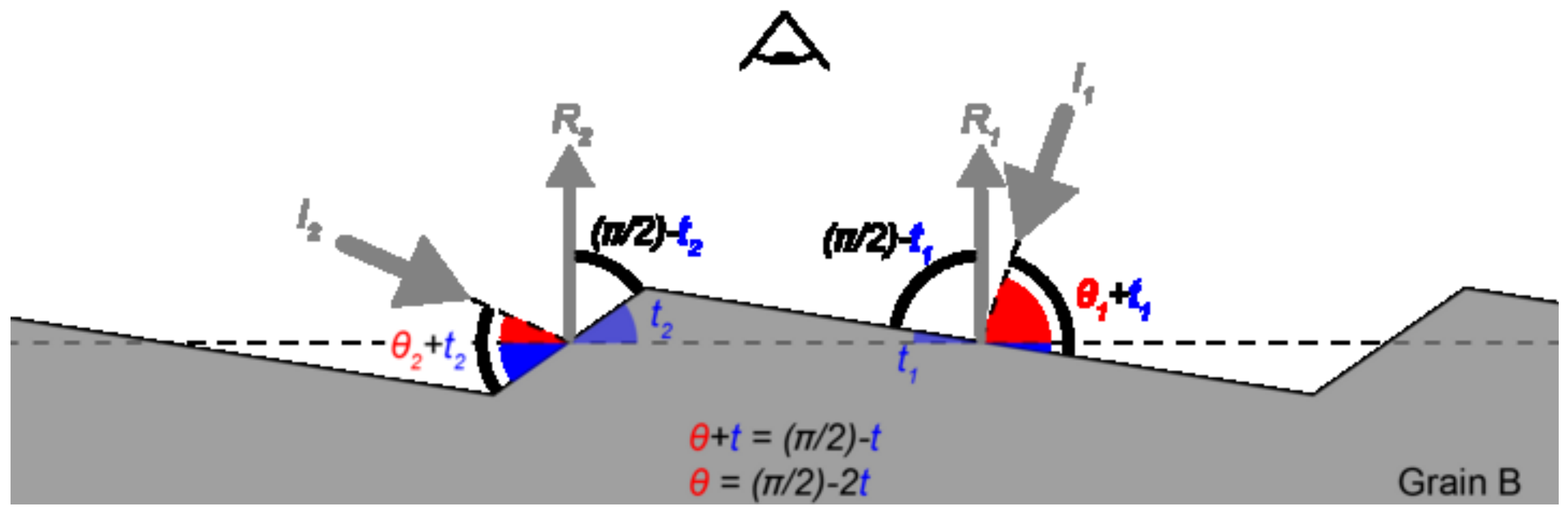




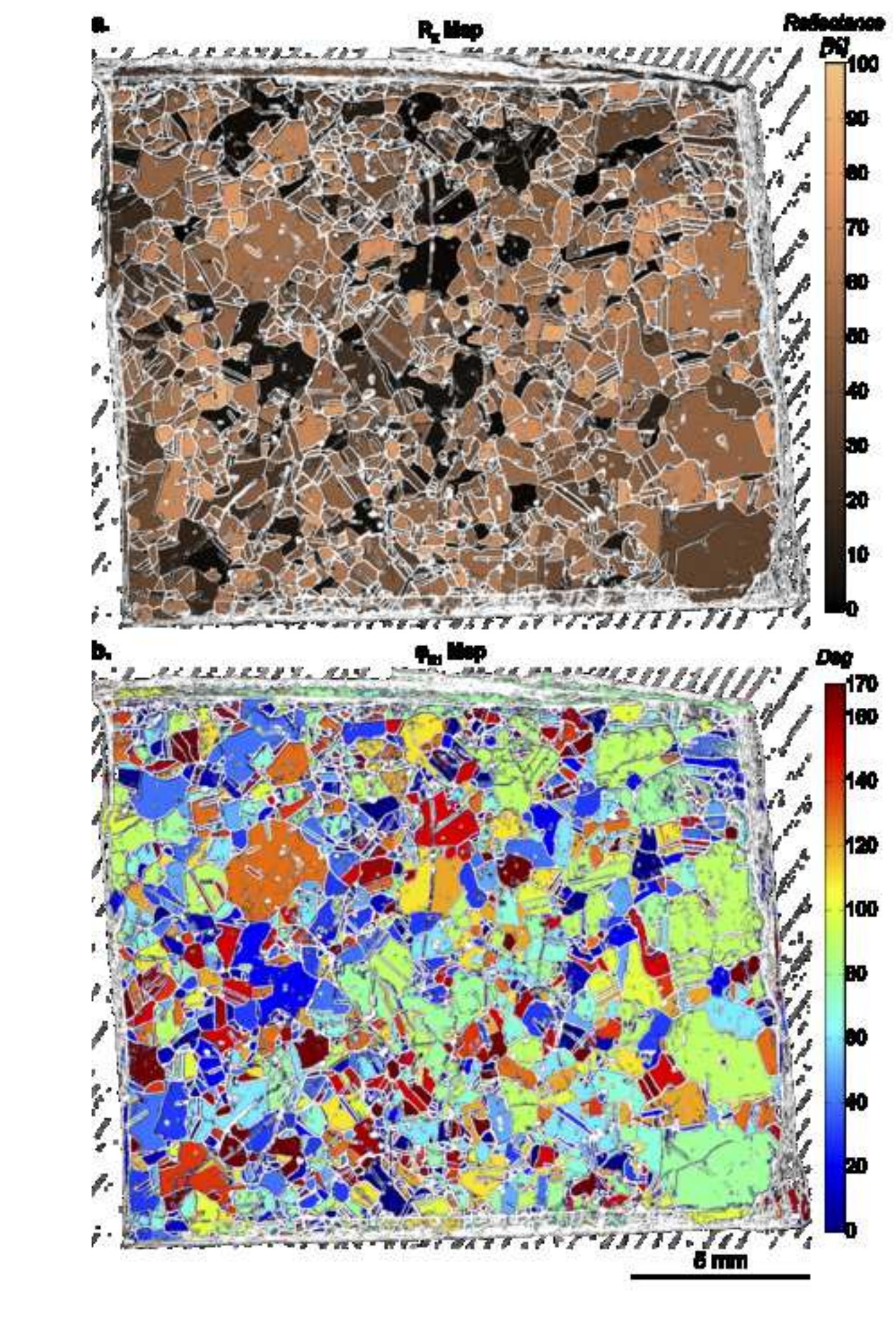

Figure(s)
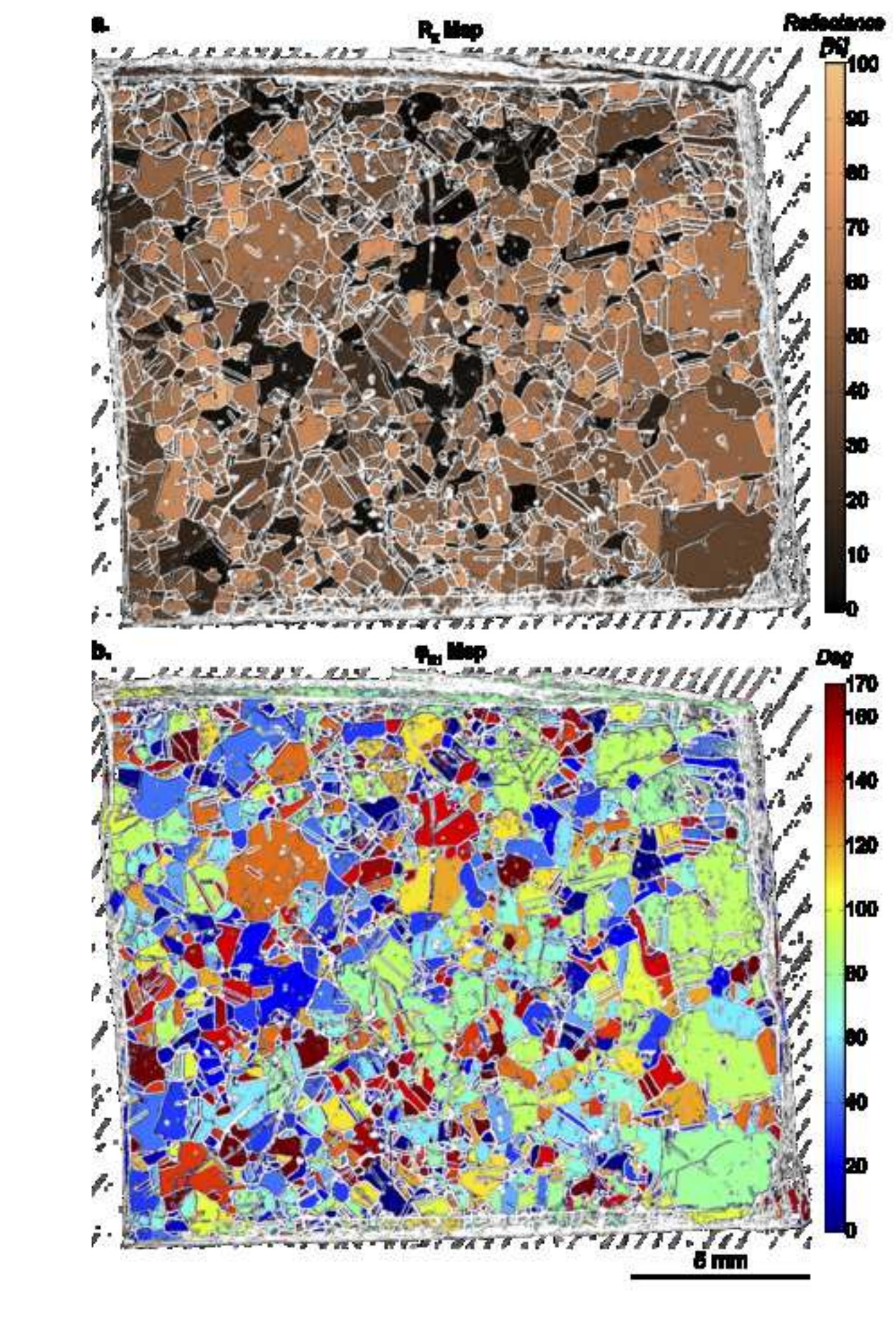

igure(s)
. 
8EI

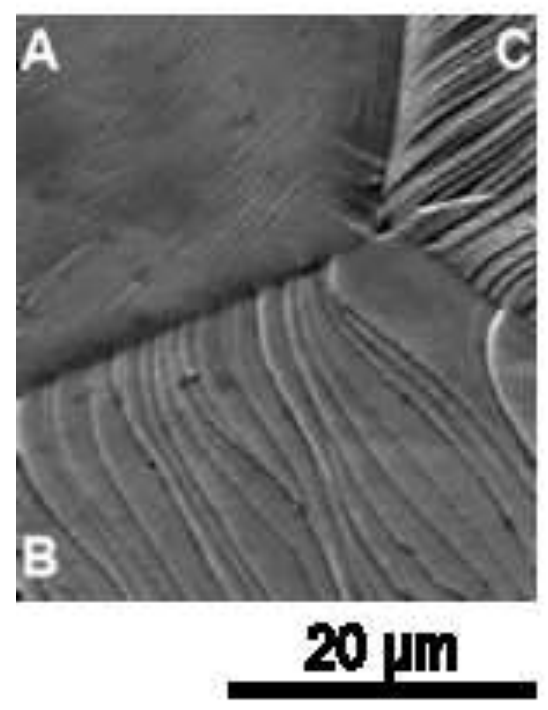

AFi

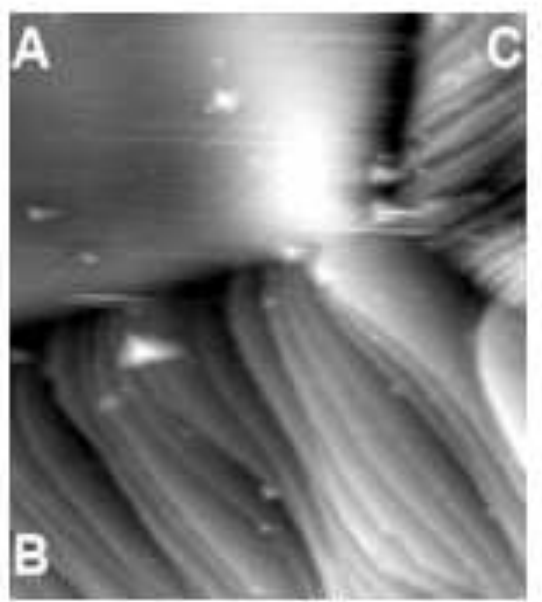

$20 \mu \mathrm{m}$
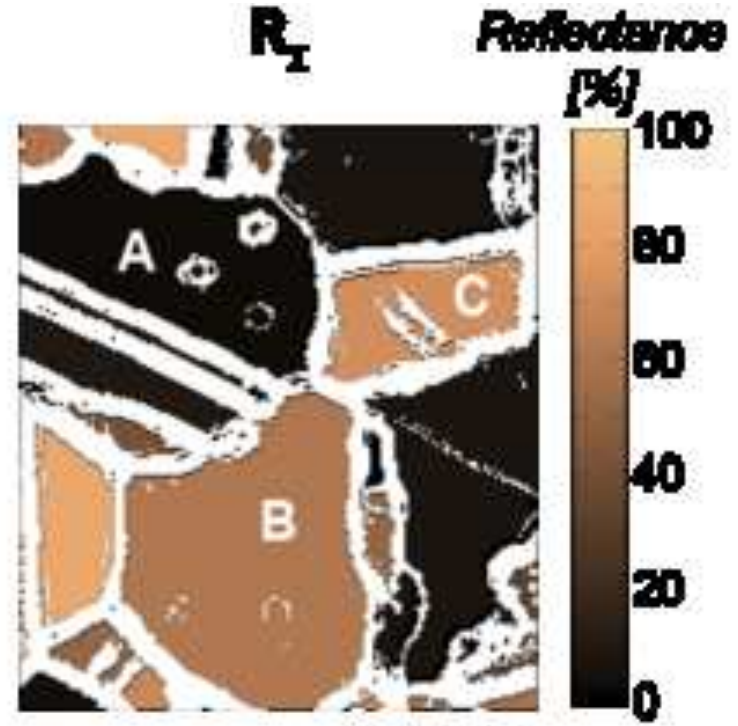

$600 \mathrm{pm}$

B

$R,-49.2 \%$

$R_{2}=27.6 \%$

$R_{\mathbf{x}}=01 \%$

$P$ Fich $=0.32 \mathrm{~mm}$

Holcht $=0.25 \mathrm{pm}$

FMs $=0.39 \mathrm{~mm}$
$R_{1}=46.8 \%$

$R=44.3 \%$

$R_{\mathbf{z}}=72 \%$
Piteh $=0.41 \mathrm{~mm}$ Holcht $=0.45 \mathrm{\mu m}$ $\mathrm{F} H \mathrm{H}=0.62 \mathrm{\mu m}$

Roughnses

Fich =0.58 pm

Hoinht $=0.19 \mathrm{pm}$

RM $=0.16$ m

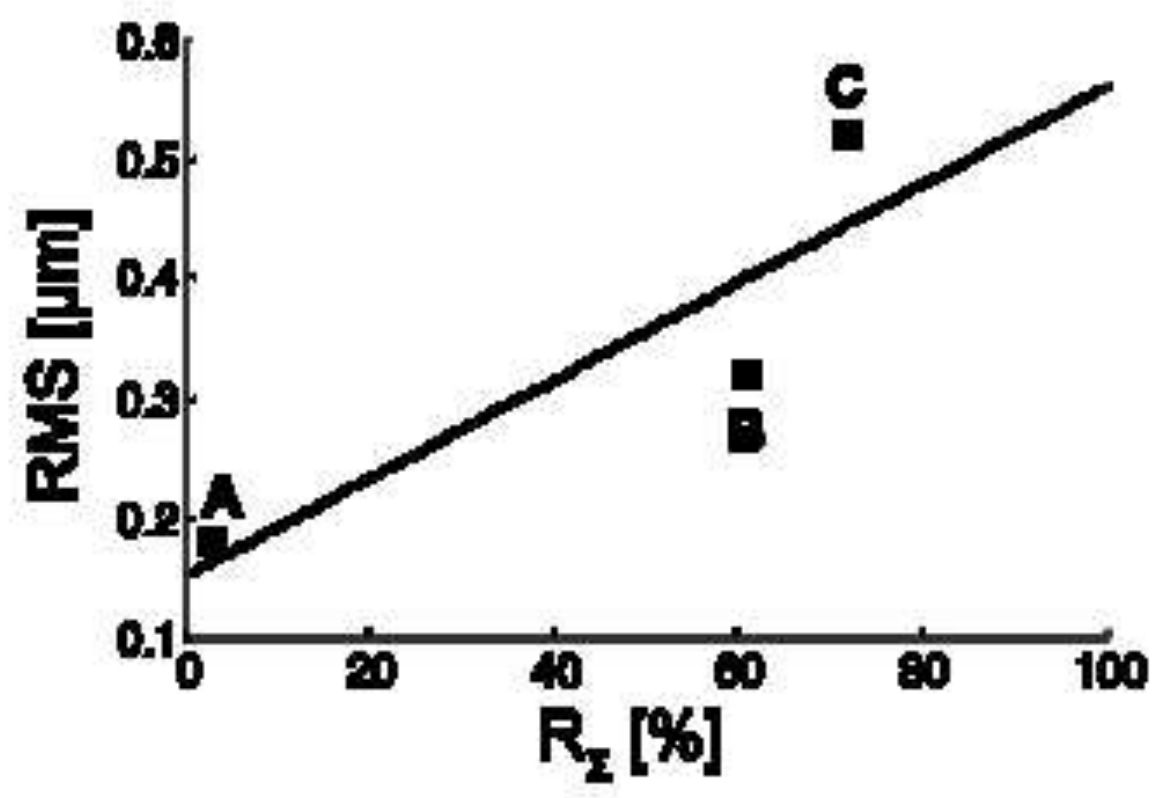




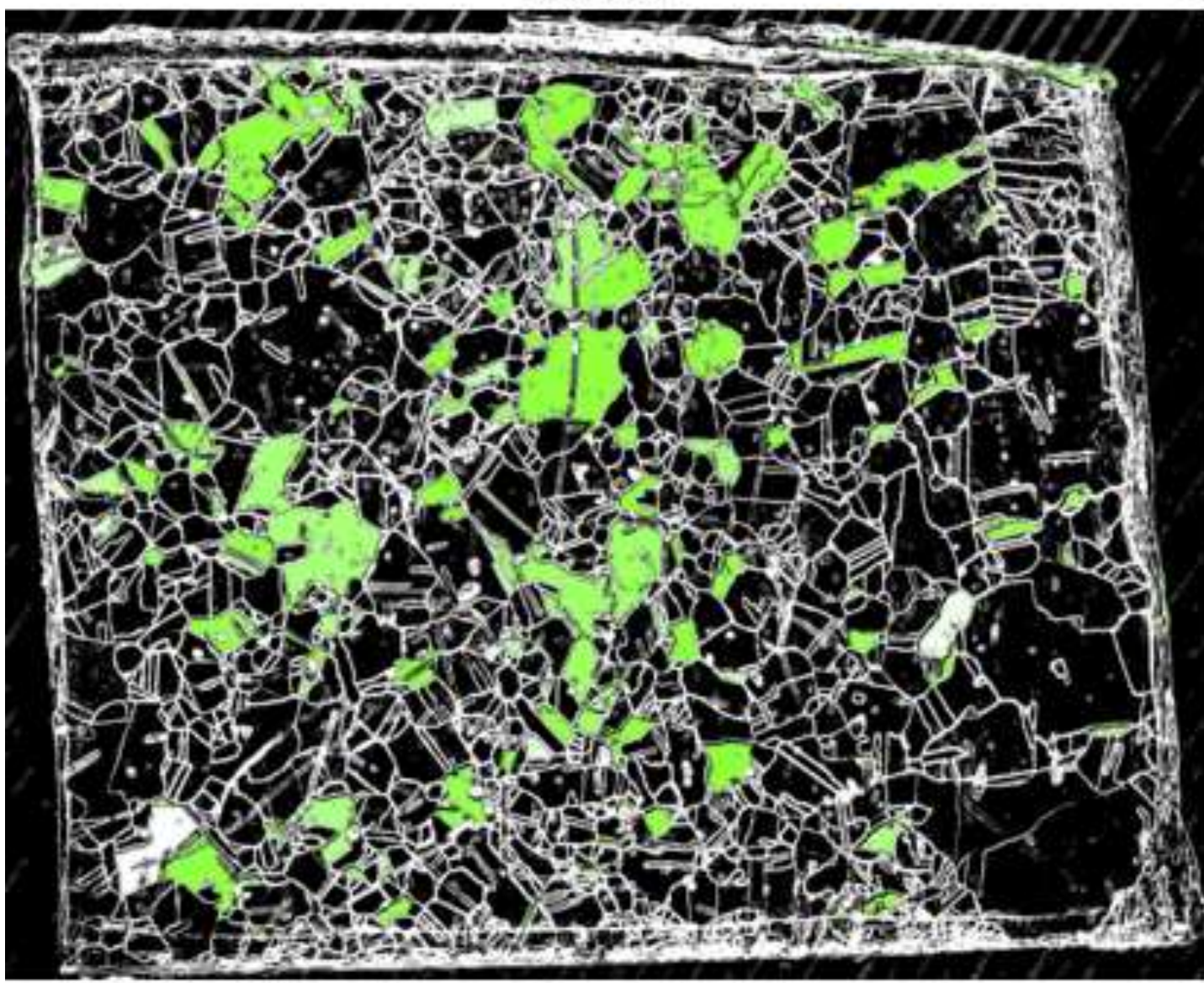

$8 \mathrm{~mm}$

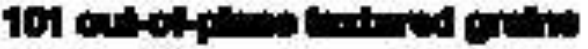

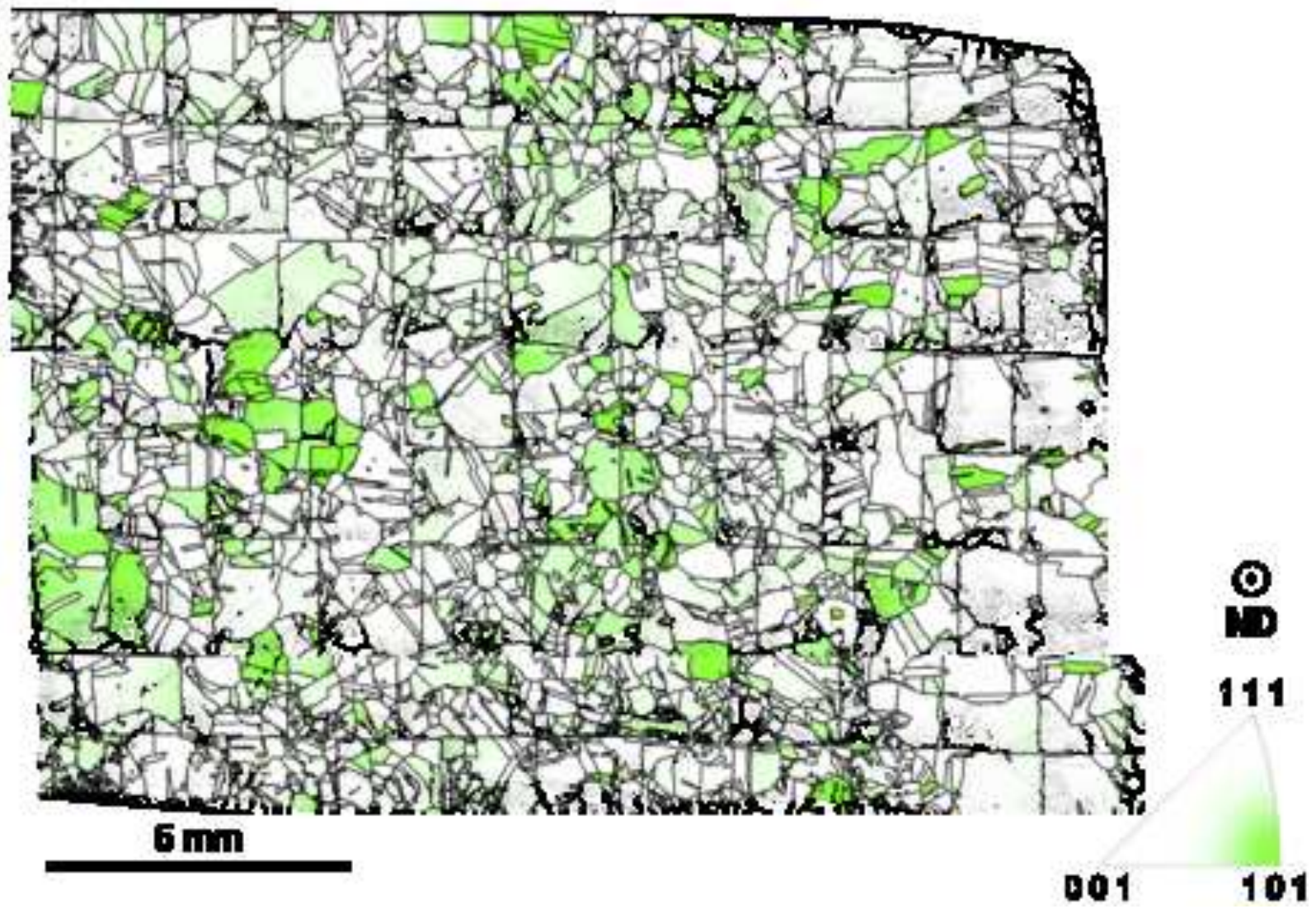




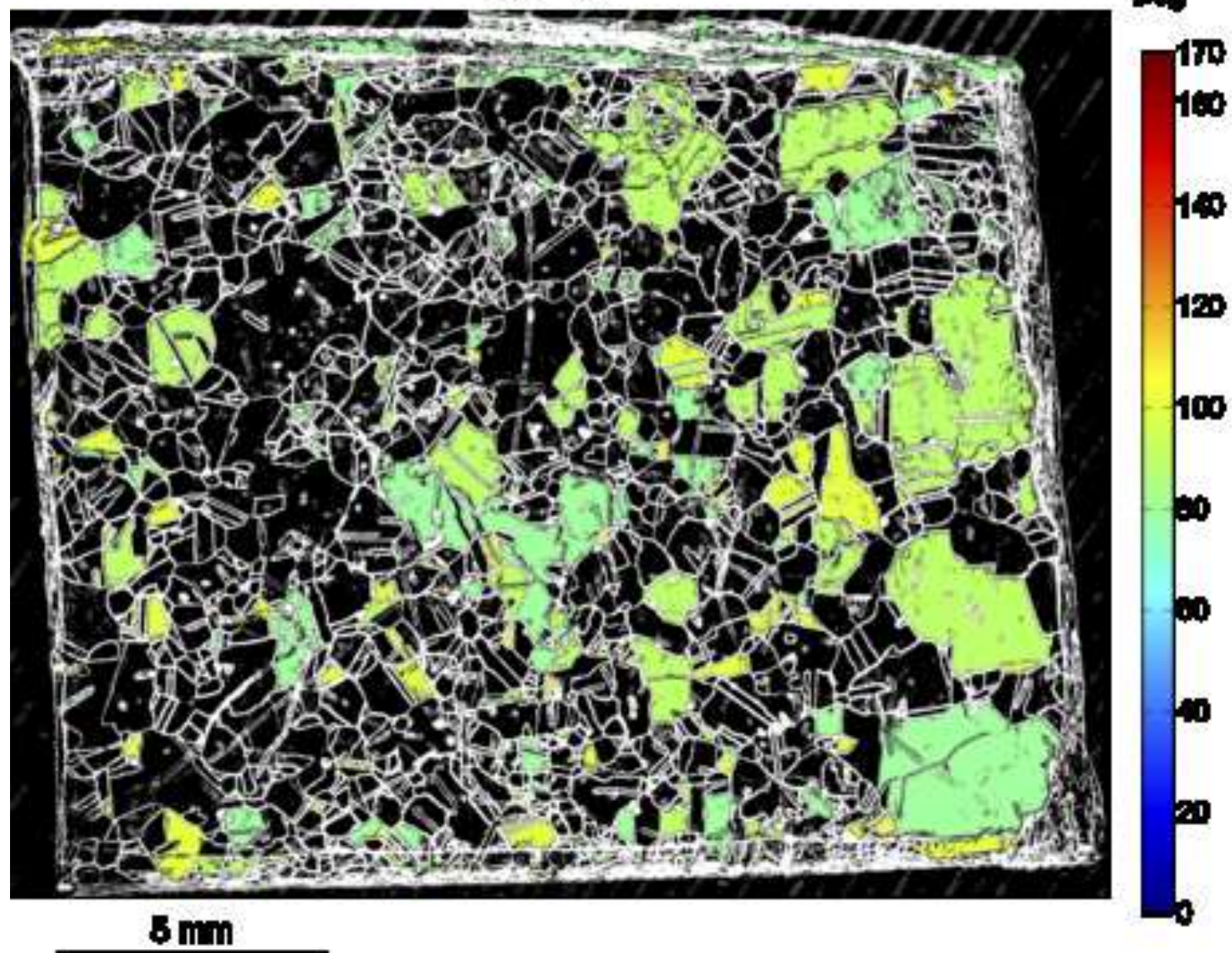

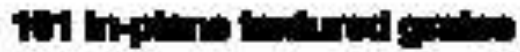

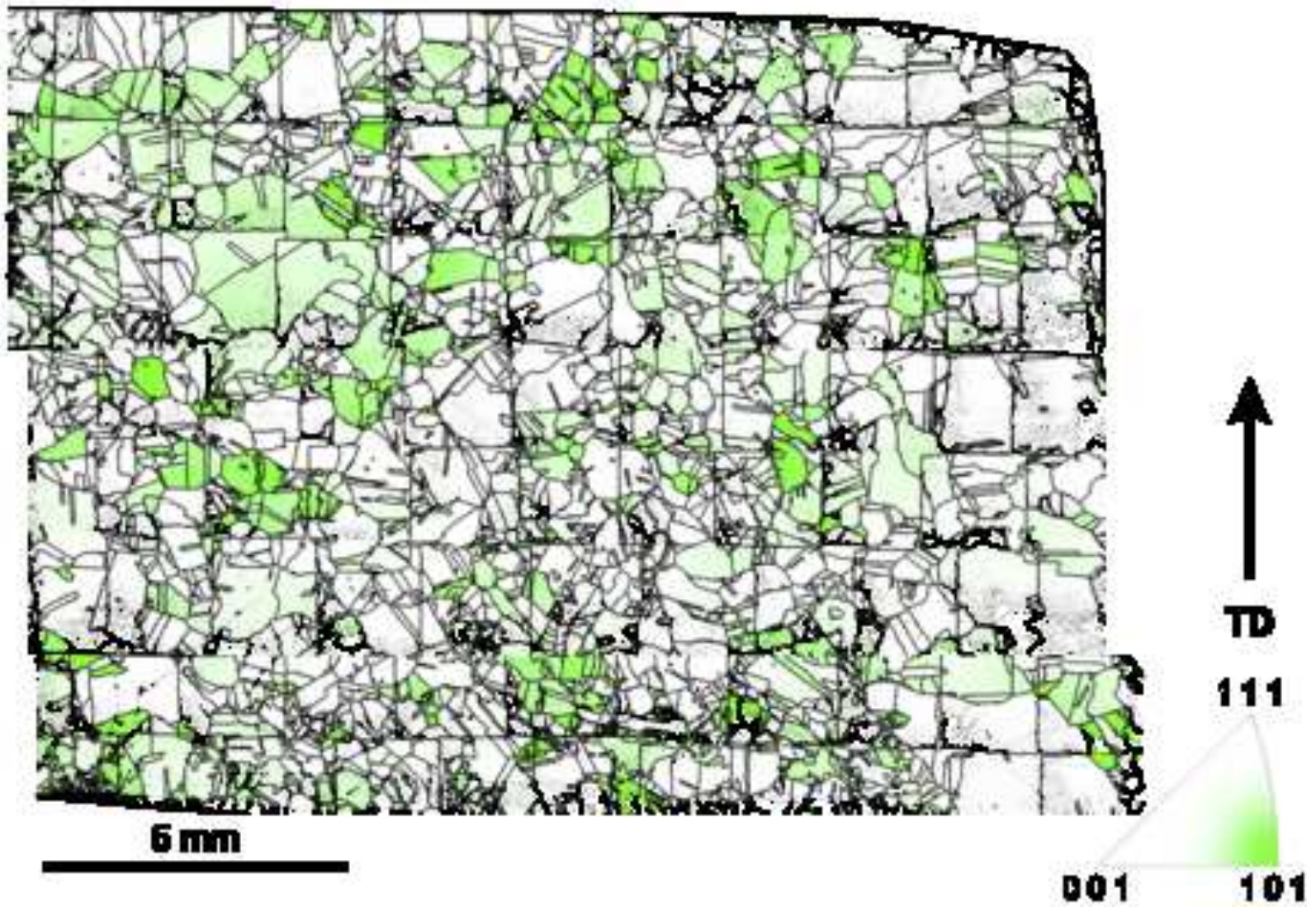


meesurements

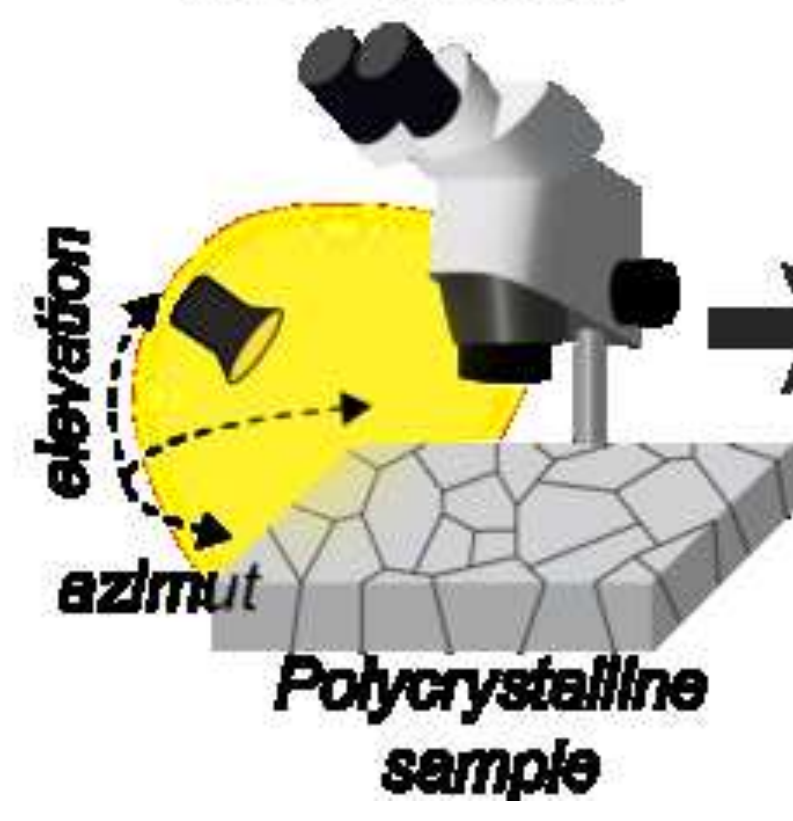

\section{Directional refiectence}

Numerical image enelysts

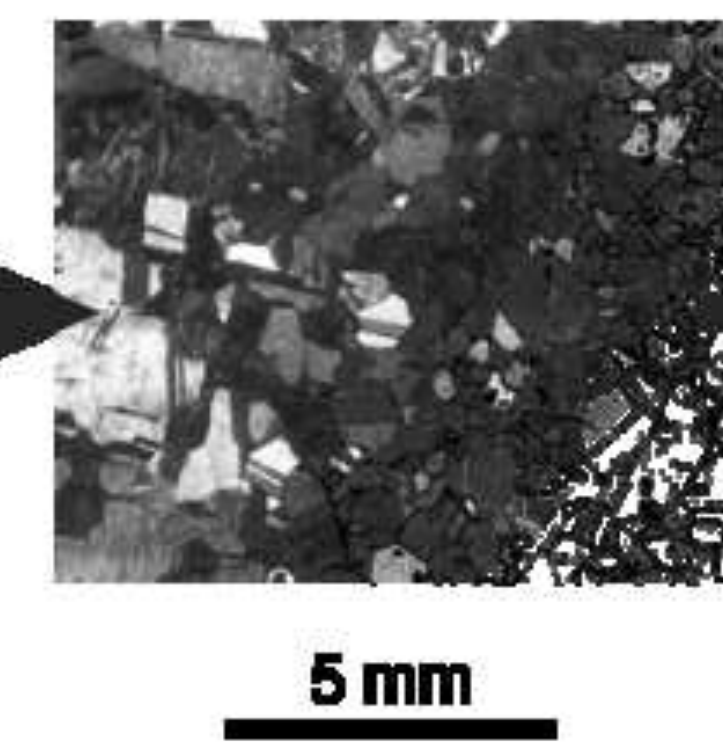

Orientation meps

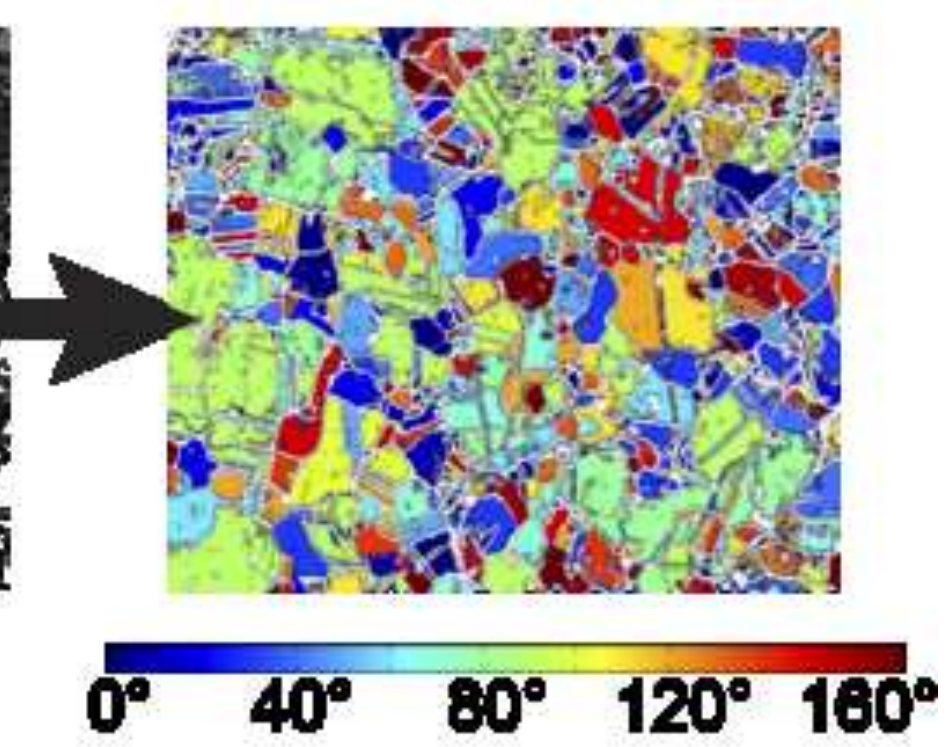

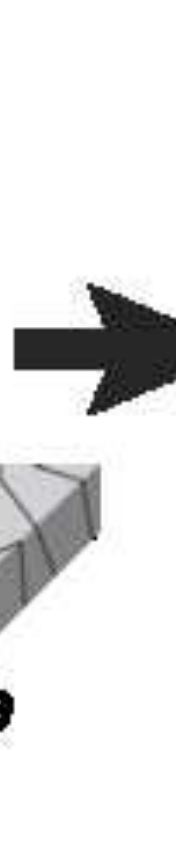

